\title{
A CERTAIN PROPERTY OF GEODESICS OF THE FAMILY OF RIEMANNIAN MANIFOLDS $O_{n}^{2}($ IX)
}

\author{
By TOMINOSUKe OTSUKI
}

\section{§ 0. Introduction.}

This is exactly a continuation of Part (VIII) ([20]) with the same title written by the present author which proved the following conjecture is true for $4.5 \leqq n \leqq 5$. He aimed at giving the proof of it for $3 \leqq n \leqq 5$ but found that the method by using computors in it was not so effective, when $n$ comes near 3 and we know that the method used until now does not work well for the proof of Conjecture $C$ for $2 \leqq n \leqq 3$ by Lemma 8.1 of Part (III) ([13]). We shall show that this conjecture is also true for $2.4 \leqq n \leqq 4.5$ in the present paper by taking a new way. As the previous ones, we shall use the numerical data obtained by means of computors in the verification. We shall also use the same notation in the previous papers ( I )-(VIII).

The period $T$ of any non-trivial solution $x(t)$ of the non-linear differential equation of order 2 :

$$
n x\left(1-x^{2}\right) \frac{d^{2} x}{d t^{2}}+\left(\frac{d x}{d t}\right)^{2}+\left(1-x^{2}\right)\left(n x^{2}-1\right)=0
$$

with a constant $n>1$ such that $x^{2}+x^{\prime 2}<1$ is given by the integral:

$$
T=\sqrt{n c} \int_{x_{0}}^{x_{1}} \frac{d x}{x \sqrt{(n-x)\left\{x(n-x)^{n-1}-c\right\}}},
$$

where $x_{0}=n\{\min x(t)\}^{2}, x_{1}=n\{\max x(t)\}^{2}, 0<x_{0}<1<x_{1}<n$ and $c=x_{0}\left(n-x_{0}\right)^{n-1}$ $=x_{1}\left(n-x_{1}\right)^{n-1}$.

Conjecture C. The period $T$ as a function of $\tau=\left(x_{1}-1\right) /(n-1)$ and $n$ is monotone decreasing with respect to $n(>2)$ for any fixed $\tau(0<\tau<1)$.

\section{$\S 1$. Preliminaries.}

Setting $T=\Omega(\tau, n)$, we have the formulas

$$
\frac{\partial \Omega(\tau, n)}{\partial n}=-\frac{\sqrt{c}}{2(B-c) n \sqrt{n}} \int_{x_{0}}^{x_{1}} \frac{(1-x) \sqrt{x(n-x)^{n-1}-c}}{x^{2}(n-x)^{n}} V\left(x, x_{1}\right) d x
$$

Received November 10, 1987 
((7.4) and Proposition 3 in (III)), where $B=(n-1)^{n-1}$ and

$$
\begin{aligned}
& V\left(x, x_{1}\right)=\frac{x^{2} \sqrt{n-x} F_{2}(x)}{(1-x)^{5}}\left\{\lambda(x)-\tilde{\lambda}\left(x_{1}\right)\right\} \\
& +\frac{X^{2} \sqrt{n-X} F_{2}(X)}{(X-1)^{5}}\left\{\lambda(X)-\tilde{\lambda}\left(x_{1}\right)\right\} \\
& -\left\{\frac{3 x^{2} f_{0}(x)}{(x-1)^{3} \sqrt{n-x}}-\frac{2 n x^{2}(B-\phi(x))}{(x-1)^{2} \sqrt{n-x}}\right\} \\
& +\left\{\frac{3 X^{2} f_{0}(X)}{(X-1)^{3} \sqrt{n-X}}-\frac{2 n X^{2}(B-\psi(X))}{(X-1)^{2} \sqrt{n-X}}\right\}, \\
& f_{0}(z):=(2 n-1-z) B-(n-z)^{n-1}\left\{n-z+(n-1) z^{2}\right\}, \\
& F_{2}(z):=-P_{2}(z) B+(n-z)^{n-1} P_{3}(z) \text {, } \\
& P_{2}(z):=(2 n+1) z^{2}-2\left(2 n^{2}+5 n-4\right) z+16 n^{2}-16 n+3 \text {, } \\
& P_{3}(z):=-(n-1) z^{3}+\left(2 n^{2}-7 n+8\right) z^{2}+(n-3)(4 n-1) z+3 n(2 n-1), \\
& \lambda(z):=\log (n-z)+\frac{n-1}{n-z}, \quad \phi(z):=z(n-z)^{n-1}, \\
& \tilde{\lambda}(z):=\lambda(z)-\frac{n}{n-1} \frac{(z-1)^{2}}{z(n-z)}=\log (n-z)+\frac{n z-1}{(n-1) z},
\end{aligned}
$$

and $X=X_{n}(x), 0<x<1<X<n$, defined by $\phi(x)=\phi(X)$.

By Lemma 8.1 in (II), we know the following:

FACT 1. For $0<x<1, V\left(x, x_{1}\right)$ is increasing with respect to $x_{1}$ in $X_{n}(x)$ $\leqq x_{1}<n$

FACT 2. We have

$$
V\left(x, X_{n}(x)\right) / B=\frac{n\left(4 n^{2}-10 n+3\right)}{(n-1)^{3}} \sqrt{n-X_{n}(x)}\left\{1+O\left(n-X_{n}(x)\right)\right\}
$$

near $x=0$, which implies $V\left(x, X_{n}(x)\right)$ is negative near $x=0$, when $2<n<\frac{5+\sqrt{13}}{4}$ $=2.151387819 \ldots((8.10)$ in $($ III $))$;

FACT 3. We have

$$
V\left(x, X_{n}(x)\right) / B=\frac{n(2 n-1)\left(n^{2}-n-3\right)}{6(n-1)^{2} \sqrt{n-1}}(1-x)\{1+O(1-x)\},
$$

near $x=1$, which implies $V\left(x, X_{n}(x)\right)$ is negative near $x=1$, when $2<n<\frac{1+\sqrt{13}}{2}$ $=2.302775638 \cdots((8.22)$ in (III)).

Since we have from (1.7) and (1.8) 


$$
\begin{aligned}
& \lambda(x)-\tilde{\lambda}\left(x_{1}\right)=\frac{n}{n-1} \frac{(x-1)^{2}}{x(n-x)}+\tilde{\lambda}(x)-\tilde{\lambda}\left(x_{1}\right), \\
& \lambda(X)-\tilde{\lambda}\left(x_{1}\right)=\frac{n}{n-1} \frac{(X-1)^{2}}{X(n-X)}+\tilde{\lambda}(X)-\tilde{\lambda}\left(x_{1}\right),
\end{aligned}
$$

introducing an auxiliary function

(1.11) $\quad \eta(z, n):=\frac{3 z^{2} f_{0}(z)}{(z-1)^{3} \sqrt{n-z}}-\frac{2 n z^{2}\{B-\psi(z)\}}{(z-1)^{2} \sqrt{n-z}}+\frac{n}{n-1} \frac{z F_{2}(z)}{(z-1)^{3} \sqrt{n-z}}$,

we obtain from (1.2)

$$
\begin{aligned}
V\left(x, x_{1}\right)= & \frac{x^{2} \sqrt{n-x} F_{2}(x)}{(1-x)^{5}}\left\{\tilde{\lambda}(x)-\tilde{\lambda}\left(x_{1}\right)\right\} \\
& +\frac{X^{2} \sqrt{n-X} F_{2}(X)}{(X-1)^{5}}\left\{\tilde{\lambda}(X)-\tilde{\lambda}\left(x_{1}\right)\right\}-\eta(x, n)+\eta(X, n),
\end{aligned}
$$

and

(1.13) $V\left(x, X_{n}(x)\right)=\frac{x^{2} \sqrt{n-x} F_{2}(x)}{(1-x)^{5}}\{\tilde{\lambda}(x)-\tilde{\lambda}(X)\}-\eta(x, n)+\eta(X, n)$.

Furthermore, we have

$$
\eta(x, n)=\frac{x}{(x-1)^{3} \sqrt{n-x}}\left[3 x f_{0}(x)-2 n x(x-1)\{B-\psi(x)\}+\frac{n}{n-1} F_{2}(x)\right]
$$

and

$$
\begin{array}{rl}
3 & x f_{0}(x)-2 n x(x-1)\{B-\psi(x)\}+\frac{n}{n-1} F_{2}(x) \\
= & 3 x(2 n-1-x) B-3 x(n-x)^{n-1}\left\{n-x+(n-1) x^{2}\right\} \\
& -2 n x(x-1)\left\{B-x(n-x)^{n-1}\right\}+\frac{n}{n-1}\left\{-P_{2}(x) B+(n-x)^{n-1} P_{3}(x)\right\} \\
= & B\left[3 x(2 n-1-x)-2 n x(x-1)-\frac{n}{n-1} P_{2}(x)\right] \\
& +(n-x)^{n-1}\left[-3 x\left\{n-x+(n-1) x^{2}\right\}+2 n x^{2}(x-1)+\frac{n}{n-1} P_{3}(x)\right] \\
= & \frac{1}{n-1}\left[-\widetilde{P}_{2}(x, n) B+(n-x)^{n-1} \tilde{P}_{3}(x, n)\right],
\end{array}
$$

where

$$
\begin{aligned}
\tilde{P}_{2}(x, n):= & \left(4 n^{2}+2 n-3\right) x^{2}-\left(4 n^{3}+18 n^{2}-19 n+3\right) x+n\left(16 n^{2}-16 n+3\right), \\
\tilde{P}_{3}(x, n):= & -(n-1)(2 n-3) x^{3}+\left(2 n^{3}-9 n^{2}+13 n-3\right) x^{2} \\
& +2 n\left(2 n^{2}-8 n+3\right) x+3 n^{2}(2 n-1),
\end{aligned}
$$

hence 


$$
\eta(x, n)=\frac{x}{(n-1)(x-1)^{3} \sqrt{n-x}}\left[-\widetilde{P}_{2}(x, n) B+(n-x)^{n-1} \tilde{P}_{3}(x, n)\right] .
$$

Since we obtain easily

$$
\tilde{P}_{2}(x, n)=(n-x) \tilde{Q}_{1}(x, n) \text { and } \tilde{P}_{3}(x, n)=(n-x) \tilde{Q}_{2}(x, n),
$$

where

$$
\tilde{Q}_{1}(x, n):=-\left(4 n^{2}+2 n-3\right) x+16 n^{2}-16 n+3 \text {, }
$$

$$
\tilde{Q}_{2}(x, n):=(n-1)(2 n-3) x^{2}+\left(4 n^{2}-10 n+3\right) x+3 n(2 n-1),
$$

we have

$$
\eta(x, n) / B=\frac{x \sqrt{n-x}}{(n-1)(x-1)^{3}}\left[-\tilde{Q}_{1}(x, n)+\left(\frac{n-x}{n-1}\right)^{n-1} \tilde{Q}_{2}(x, n)\right] .
$$

Our principle of the verification of Conjecture $C$, this time, is that investigating the behavior of $\eta(x, n)$, we shall show that

$$
\eta\left(X_{n}(x), n\right)-\eta(x, n)>0 \quad \text { for } 0<x<1
$$

with $n>\frac{5+\sqrt{13}}{4}+0.011$ and using the properties of the positive function $\frac{x^{2} \sqrt{n-x} F_{2}(x)}{(1-x)^{5}}\left\{\tilde{\lambda}\left(X_{n}(x)\right)-\tilde{\lambda}(x)\right\}$ in $0<x<1$, we shall show that $V\left(x, X_{n}(x)\right)>0$ for $0<x<1$ with $n \geqq 2.4$. Then by Fact 1 , we see that

$$
V\left(x, x_{1}\right)>0 \text { for } 0<x<1, X_{n}(x) \leqq x_{1}<n,
$$

which implies

$$
\frac{\partial \Omega(\tau, n)}{\partial n}<0 \text { for } n \geqq 2.4 \text { with } 0<\tau<1 \text {. }
$$

\section{$\S 2$. Properties of $\eta(x, n)(0 \leqq x \leqq n)$.}

In order to guess the behavior of $\eta(x, n)$, we show here the graphs of $\eta(x, 2)$ and $\eta(x, 3)$ as examples.

i) $\eta(x, 2)=-x \sqrt{2-x}$.

$$
X=2-x \text {, }
$$$$
\eta(X, 2)-\eta(x, 2)=-\sqrt{x(2-x)}(\sqrt{2-x}-\sqrt{x})<0 \text { for } 0<x<1 \text {. }
$$

ii) $\eta(x, 3)=\frac{3}{2} x(2 x-3) \sqrt{3-x}, \quad \eta^{\prime}(x, 3)=-\frac{3\left(10 x^{2}-33 x+18\right)}{4 \sqrt{3-x}}$,

$$
\eta(0)=\eta(3 / 2)=\eta(3)=0, \quad \eta(1)=-\frac{3 \sqrt{2}}{2},
$$




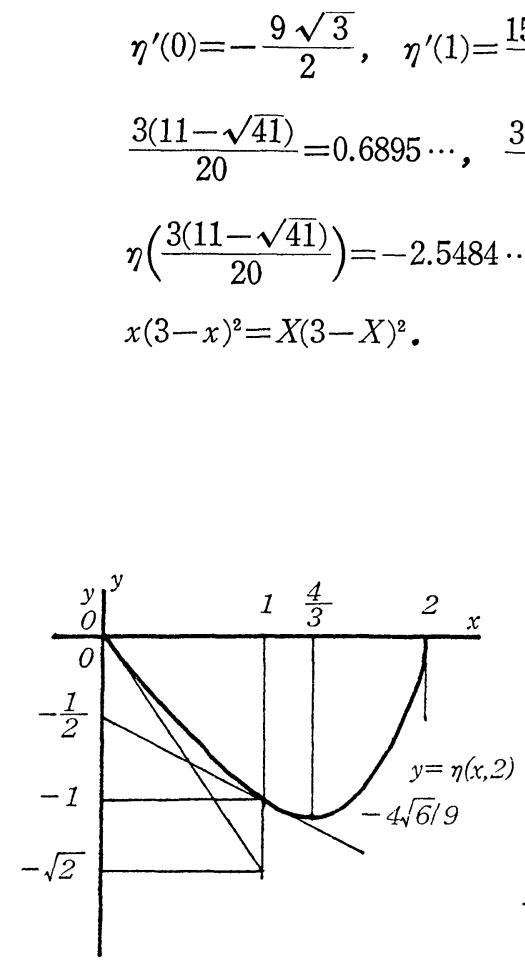

Fig. 1.

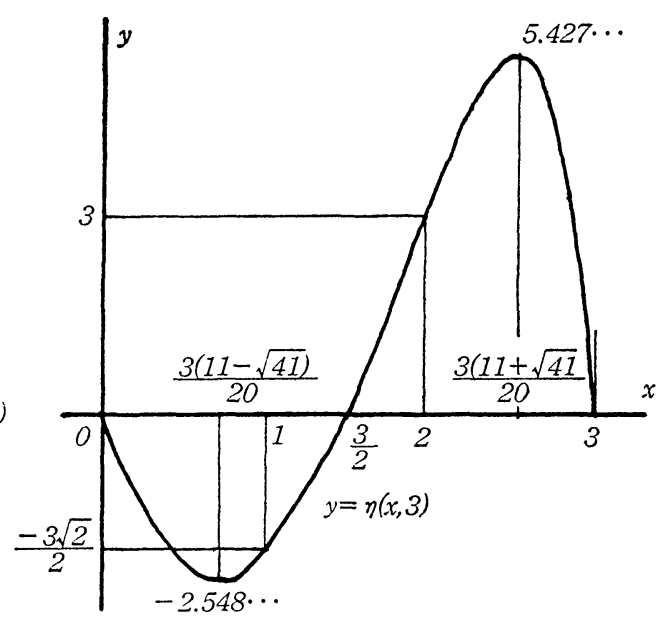

Fig. 2.

We may imagine as

$$
\eta(X, 3)-\eta(x, 3)>0
$$

for $0<x<1$ at this stage.

\section{LEMMA 2.1. We have}

$$
\frac{d}{d x} \eta(x, n) / B=\frac{1}{2(n-1)(x-1)^{4} \sqrt{n-x}}\left[\tilde{Q}_{3}(x, n)-\left(\frac{n-x}{n-1}\right)^{n-1} \tilde{Q}_{4}(x, n)\right],
$$

where

$$
\begin{aligned}
\tilde{Q}_{3}(x, n):= & \left(4 n^{2}+2 n-3\right) x^{3}-8(n-1)\left(n^{2}+5 n-3\right) x^{2} \\
& +3\left(16 n^{3}-40 n^{2}+24 n-3\right) x+2 n(4 n-1)(4 n-3), \\
\tilde{Q}_{4}(x, n):= & (n-1)(2 n-1)(2 n-3) x^{4}+(2 n-3)\left(2 n^{2}-13 n+8\right) x^{3} \\
& +3\left(8 n^{3}-26 n^{2}+21 n-3\right) x^{2}+n\left(28 n^{2}-52 n+15\right) x+6 n^{2}(2 n-1) .
\end{aligned}
$$

Proof. From (1.16), we have 


$$
\begin{aligned}
& \frac{d}{d x} \eta(x, n) / B \\
& =\frac{3 x^{2}-(4 n-3) x-2 n}{2(n-1)(x-1)^{4} \sqrt{n-x}}\left[-\tilde{Q}_{1}+\left(\frac{n-x}{n-1}\right)^{n-1} \tilde{Q}_{2}\right] \\
& +\frac{x \sqrt{n-x}}{(n-1)(x-1)^{3}}\left[-\tilde{Q}_{1}^{\prime}+\left(\frac{n-x}{n-1}\right)^{n-1} \tilde{Q}_{2}^{\prime}-\left(\frac{n-x}{n-1}\right)^{n-2} \tilde{Q}_{2}\right] \\
& =\frac{1}{2(n-1)(x-1)^{4} \sqrt{n-x}}\left[-\left(3 x^{2}-(4 n-3) x-2 n\right) \tilde{Q}_{1}-2 x(x-1)(n-x) \tilde{Q}_{1}^{\prime}\right. \\
& \quad+\left(\frac{n-x}{n-1}\right)^{n-1}\left\{\left(3 x^{2}-(4 n-3) x-2 n\right) \tilde{Q}_{2}\right. \\
& \left.\left.\quad+2 x(x-1)(n-x) \tilde{Q}_{2}^{\prime}-2(n-1) x(x-1) \tilde{Q}_{2}\right\}\right] .
\end{aligned}
$$

Computing separately the terms in the brackets, we have

$$
\begin{aligned}
- & \left(3 x^{2}-(4 n-3) x-2 n\right) \tilde{Q}_{1}-2 x(x-1)(n-x) \tilde{Q}_{1}^{\prime} \\
= & \left(3 x^{2}-(4 n-3) x-2 n\right)\left\{\left(4 n^{2}+2 n-3\right) x-\left(16 n^{2}-16 n+3\right)\right\} \\
& +2 x(x-1)(n-x)\left(4 n^{2}+2 n-3\right) \\
= & \left(4 n^{2}+2 n-3\right) x^{3}-8(n-1)\left(n^{2}+5 n-3\right) x^{2} \\
& +3\left(16 n^{3}-40 n^{2}+24 n-3\right) x+2 n(4 n-1)(4 n-3)=\tilde{Q}_{3}(x, n)
\end{aligned}
$$

and

$$
\begin{aligned}
- & \left(3 x^{2}-(4 n-3) x-2 n\right) \tilde{Q}_{2}-2 x(x-1)(n-x) \tilde{Q}_{2}^{\prime}+2(n-1) x(x-1) \tilde{Q}_{2} \\
= & \left((2 n-5) x^{2}+(2 n-1) x+2 n\right)\left\{(n-1)(2 n-3) x^{2}+\left(4 n^{2}-10 n+3\right) x+3 n(2 n-1)\right\} \\
& +2 x\left(x^{2}-(n+1) x+n\right)\left\{2(n-1)(2 n-3) x+4 n^{2}-10 n+3\right\} \\
= & (n-1)(2 n-1)(2 n-3) x^{4}+(2 n-3)\left(2 n^{2}-13 n+8\right) x^{3} \\
& +3\left(8 n^{3}-26 n^{2}+21 n-3\right) x^{2}+n\left(28 n^{2}-52 n+15\right) x+6 n^{2}(2 n-1)=\tilde{Q}_{4}(x, n),
\end{aligned}
$$

from which we obtain (2.1).

Q.E.D.

LEMMA 2.2. We have
i) $\eta(0, n)=\eta(n, n)=0$,
ii) $\lim _{x \rightarrow 1} \eta(x, n)=-\frac{1}{2} n(n-1)^{n-2.5}$,
iii) $\eta^{\prime}(0, n)=(n-1)^{n-2} \sqrt{n}\left\{(4 n-1)(4 n-3)-3 e_{n-1} n(2 n-1)\right\}<0$
for $n \geqq 2$, where $e_{n-1}=\left(1+\frac{1}{n-1}\right)^{n-1}$,

iv) $\lim _{x \rightarrow n-0} \eta^{\prime}(x, n)=-\infty$ for $n>\frac{5+\sqrt{13}}{4}$, 
v) $\lim _{x \rightarrow 1} \eta^{\prime}(x, n)=\frac{1}{12} n(2 n-1)\left(n^{2}-n-3\right)(n-1)^{n-3.5}>0$ for $n>\frac{1+\sqrt{13}}{2}$ and $<0$ for $2 \leqq n<\frac{1+\sqrt{13}}{2}$.

Proof. i) is evident from (1.16). In order to prove ii) we set $x=1+y$. From (1.14) and (1.15) we have

$$
\begin{aligned}
& \tilde{Q}_{1}(1+y, n)=-\left(4 n^{2}+2 n-3\right) y+6(n-1)(2 n-1), \\
& \tilde{Q}_{2}(1+y, n)=(n-1)(2 n-3) y^{2}+\left(8 n^{2}-20 n+9\right) y+6(n-1)(2 n-1)
\end{aligned}
$$

and

$$
\left(\frac{n-x}{n-1}\right)^{n-1}=\left(1-\frac{y}{n-1}\right)^{n-1}=1-y+\frac{n-2}{2(n-1)} y^{2}-\frac{(n-2)(n-3)}{6(n-1)^{2}} y^{3}+\cdots,
$$

from which we obtain easily

$$
-\tilde{Q}_{1}(1+y, n)+\left(1-\frac{y}{n-1}\right)^{n-1} \tilde{Q}_{2}(1+y, n)=-\frac{n}{2(n-1)} y^{3}+O\left(y^{4}\right)
$$

near $y=0$. Hence we obtain from (1.16)

$$
\lim _{x \rightarrow 1} \eta(x, n)=-(n-1)^{n-2} \sqrt{n-1} \frac{n}{2(n-1)}=-\frac{n(n-1)^{n-2.5}}{2} .
$$

Regarding iii), we have easily from (2.1)

$$
\begin{aligned}
\eta^{\prime}(0, n) & =\frac{(n-1)^{n-2}}{2 \sqrt{n}}\left\{2 n(4 n-1)(4 n-3)-e_{n-1} 6 n^{2}(2 n-1)\right\} \\
& =(n-1)^{n-2} \sqrt{n}\left\{(4 n-1)(4 n-3)-3 e_{n-1} n(2 n-1)\right\} .
\end{aligned}
$$

In order to prove the negativeness of this quantity for $n \geqq 2$, we show that

$$
\frac{3 n(2 n-1)}{(4 n-1)(4 n-3)}\left(\frac{n}{n-1}\right)^{n-1} \searrow \text { in } 2<n<+\infty \text {. }
$$

We have

$$
\begin{aligned}
& \frac{d}{d n} \log \left\{\frac{3 n(2 n-1)}{(4 n-1)(4 n-3)}\left(\frac{n}{n-1}\right)^{n-1}\right\} \\
& =\left(\frac{1}{n}+\frac{2}{2 n-1}-\frac{4}{4 n-1}-\frac{4}{4 n-3}\right)+\left(\log \frac{n}{n-1}-\frac{1}{n}\right) \\
& =\frac{2}{2 n-1}-\frac{4}{4 n-1}-\frac{4}{4 n-3}+\log \frac{n}{n-1} \rightarrow 0 \quad(\text { as } n \rightarrow+\infty),
\end{aligned}
$$

and 


$$
\begin{aligned}
& \frac{d^{2}}{d n^{2}} \log \left\{\frac{3 n(2 n-1)}{(4 n-1)(4 n-3)}\left(\frac{n}{n-1}\right)^{n-1}\right\} \\
& =-\frac{4}{(2 n-1)^{2}}+\frac{16}{(4 n-1)^{2}}+\frac{16}{(4 n-3)^{2}}-\frac{1}{n(n-1)} \\
& =-\frac{8 n^{2}-8 n+1}{n(n-1)(2 n-1)^{2}}+\frac{16\left(32 n^{2}-32 n+10\right)}{\left(16 n^{2}-16 n+3\right)^{2}}\left(N=n^{2}-n\right) \\
& =-\frac{8 N+1}{N(4 N+1)}+\frac{16(32 N+10)}{(16 N+3)^{2}}=\frac{128 N^{2}-8 N-9}{N(4 N+1)(16 N+3)^{2}}>0 .
\end{aligned}
$$

which implies

$$
\frac{d}{d n}\left\{\frac{3 n(2 n-1)}{(4 n-1)(4 n-3)}\left(\frac{n}{n-1}\right)^{n-1}\right\}<0 \text { for } n \geqq 2 .
$$

and

$$
\frac{3 n(2 n-1)}{(4 n-1)(4 n-3)}\left(\frac{n}{n-1}\right)^{n-1} \searrow \text { in } 2<n<+\infty
$$

Using this fact, we obtain

$$
\frac{3 n(2 n-1)}{(4 n-1)(4 n-3)}\left(\frac{n}{n-1}\right)^{n-1}>3 e / 8=1.0193556 \cdots>1,
$$

which implies the inequality of iii).

Next, from $(2.1)$ and $\widetilde{Q}_{3}(n, n)=-n(n-1)^{2}\left(4 n^{2}-10 n+3\right)$ we obtain

$$
\eta^{\prime}(x, n)=-\frac{B n\left(4 n^{2}-10 n+3\right)}{2(n-1)^{3} \sqrt{n-i}}\{1+O(n-x)\}
$$

near $x=n$, which implies iv).

Finally, setting again $x=1+y$, from (2.2) and (2.3) we have

$$
\begin{aligned}
\tilde{Q}_{3}(1+y, n)= & \left(4 n^{2}+2 n-3\right) y^{3}-\left(8 n^{3}+20 n^{2}-70 n+33\right) y^{2} \\
& +\left(32 n^{3}-172 n^{2}+206 n-66\right) y+72 n^{3}-180 n^{2}+144 n-36, \\
\tilde{Q}_{4}(1+y, n)= & (n-1)(2 n-1)(2 n-3) y^{4} \\
& +(2 n-3)\left(10 n^{2}-25 n+12\right) y^{3}+3\left(20 n^{3}-82 n^{2}+98 n-33\right) y^{2} \\
& +2(n-1)\left(52 n^{2}-124 n+51\right) y+36(n-1)^{2}(2 n-1),
\end{aligned}
$$

from which we obtain

$$
\tilde{Q}_{3}(1+y, n)-\left(1-\frac{y}{n-1}\right)^{n-1} \tilde{Q}_{4}(1+y, n)=\frac{n(2 n-1)\left(n^{2}-n-3\right)}{6(n-1)} y^{4}+O\left(y^{5}\right)
$$

near $y=0$. Hence we obtain from (2.1) 


$$
\begin{aligned}
\lim _{x \rightarrow 1} \eta^{\prime}(x, n) & =\lim _{y \rightarrow 0} \frac{B}{2(n-1) \sqrt{n-1-y}}\left\{\frac{n(2 n-1)\left(n^{2}-n-3\right)}{6(n-1)}+O(y)\right\}, \\
& =\frac{1}{12} n(2 n-1)\left(n^{2}-n-3\right)(n-1)^{n-3.5},
\end{aligned}
$$

which is positive for $n>\frac{1+\sqrt{13}}{2}$ and negative for $2 \leqq n<\frac{1+\sqrt{13}}{2}$. Q.E.D.

Remark. From Lemma 2.2 we see that $\eta(z, n)$ and $\eta^{\prime}(z, n)$ are complex analytic with a removable singular point at $z=1$ and regular on a domain containing the real half line $x<n$.

LEMMA 2.3. When $n \geqq 2$, we have

i) $\tilde{Q}_{2}(x, n)>0$ for $-\infty<x<+\infty$,

ii) $\widetilde{Q}_{4}(x, n)>0$ for $0 \leqq x<+\infty$.

Proof. First, from (1.15) the discriminant of $\tilde{Q}_{2}(x, n)$ as a quadratic polynomial of $x$ is

$$
\left(4 n^{2}-10 n+3\right)^{2}-12 n(n-1)(2 n-1)(2 n-3)=-\left(32 n^{4}-64 n^{3}+8 n^{2}+24 n-9\right)
$$

and

$$
32 n^{4}-64 n^{3}+8 n^{2}+24 n-9 \geqq 8 n^{2}+24 n-9>0 \text { for } n \geqq 2,
$$

which implies i).

Second, we set $x=n t$ and $\widetilde{S}_{4}(t, n)=\widetilde{Q}_{4}(n t, n) / n^{2}$, then we have

$$
\begin{aligned}
\tilde{S}_{4}(t, n)= & n^{2}(n-1)(2 n-1)(2 n-3) t^{4}+n(2 n-3)\left(2 n^{2}-13 n+8\right) t^{3} \\
& +3\left(8 n^{3}-26 n^{2}+21 n-3\right) t^{2}+\left(28 n^{2}-52 n+15\right) t+6(2 n-1) .
\end{aligned}
$$

Putting $n=2$, we have

$$
\widetilde{S}_{4}(t, 2)=12 t^{4}-20 t^{3}-3 t^{2}+23 t+18
$$

and

$$
\begin{aligned}
& \left(12 t^{4}-20 t^{3}-3 t^{2}\right)^{\prime \prime}=6\left(24 t^{2}-20 t-1\right), \\
& \left(12 t^{4}-20 t^{3}-3 t^{2}+23 t\right)^{\prime}=48 t^{3}-60 t^{2}-6 t+23 \\
& =\left(24 t^{2}-20 t-1\right)\left(2 t-\frac{5}{6}\right)-\frac{62}{3} t+\frac{133}{6} \\
& \geqq-\frac{62}{3} \times \frac{5+\sqrt{31}}{12}+\frac{133}{6} \\
& =\frac{1}{18}(244-31 \sqrt{31})>0 \text { for } t \geqq 0 .
\end{aligned}
$$

Hence

$$
12 t^{4}-20 t^{3}-3 t^{2}+23 t+18 t \nearrow \text { in } 0 \leqq t<+\infty
$$


and so we obtain

$$
\widetilde{S}_{4}(t, 2) \geqq 18 \text { for } t \geqq 0 \text {. }
$$

Then, we have

$$
\begin{aligned}
\frac{\partial \widetilde{S}_{4}(t, n)}{\partial n}= & \left(20 n^{4}-48 n^{3}+33 n^{2}-6 n\right) t^{4}+\left(16 n^{3}-96 n^{2}+110 n-24\right) t^{3} \\
& +3\left(24 n^{2}-52 n+21\right) t^{2}+4(14 n-13) t+12
\end{aligned}
$$

(setting $n=m+2$ )

$$
\begin{aligned}
= & \left(20 m^{4}+112 m^{3}+225 m^{2}+190 m+56\right) t^{4}+\left(16 m^{3}-82 m-60\right) t^{3} \\
& +3\left(24 m^{2}+44 m+13\right) t^{2}+4(14 m+15) t+12 \\
= & m t\left[\left(20 m^{3}+112 m^{2}+225 m+190\right) t^{3}+2\left(8 m^{2}-41\right) t^{2}\right. \\
& +56 t^{4}-60 t^{3}+39 t^{2}+60 t+12 . \\
& +12(6 m+11) t+56]
\end{aligned}
$$

On the other hand, we see that

$$
\left(56 t^{4}-60 t^{3}+39 t^{2}\right)^{\prime \prime}=6\left(112 t^{2}-60 t+13\right)>0 \text { for }-\infty<t<+\infty
$$

and so

$$
\left(56 t^{4}-60 t^{3}+39 t^{2}+60 t\right)^{\prime}=224 t^{3}-180 t^{2}+78 t+60 \nearrow
$$

in $-\infty<t<+\infty$ and $\geqq 60$ for $t \geqq 0$. Hence we obtain

$$
56 t^{4}-60 t^{3}+39 t^{2}+60 t+12 \geqq 12 \text { for } t \geqq 0 \text {. }
$$

Next we have

$$
\begin{aligned}
& \left(20 m^{3}+112 m^{2}+225 m+190\right) t^{3}+2\left(8 m^{2}-41\right) t^{2}+12(6 m+11) t+56 \\
& =m t\left[\left(20 m^{2}+112 m+225\right) t^{2}+16 m t+72\right]+190 t^{3}-82 t^{2}+132 t+56 .
\end{aligned}
$$

We see that

and so

$$
\left(190 t^{3}-82 t^{2}+132 t\right)^{\prime}=570 t^{2}-164 t+132>0 \text { for }-\infty<t<+\infty
$$

$$
190 t^{3}-82 t^{2}+132 t+56 \geqq 56 \text { for } t \geqq 0 \text {. }
$$

Hence we see that

$$
\begin{array}{r}
\left(20 m^{3}+112 m^{2}+225 m+190\right) t^{3}+2\left(8 m^{2}-41\right) t^{2}+12(6 m+11) t+56>0 \\
\text { for } m \geqq 0 \text { and } t \geqq 0 .
\end{array}
$$

From these evaluations, we obtain

$$
\frac{\partial \tilde{S}_{4}(t, n)}{\partial n}>0 \text { for } t \geqq 0 \text { and } n \geqq 2 \text {, }
$$

and hence 


$$
\widetilde{S}_{4}(t, n)>0 \text { for } t \geqq 0 \text { and } n \geqq 2 \text { Q.E.D. }
$$

PROPOSITION 1. When $n>\frac{1+\sqrt{13}}{2}, \eta^{\prime}(x, n)=0(0<x<n)$ has two solutions $\alpha$ and $\beta$ such that $0<\alpha<1$ and $1<\beta<n$ and $\eta(x, n)$ takes its minimum at $\alpha$ and its maximum at $\beta$ and is monotone in $0<x<\alpha, \alpha<x<\beta$ and $\beta<x<n$, respectively.

Proof. By means of Lemma 2.2, it is clear that $\eta^{\prime}(x, n)=0$ has roots in each interval $0<x<1$ and $1<x<n$, when $n>\frac{1+\sqrt{13}}{2}$. Let $\alpha$ be the minimum one and $\beta$ the maximum one. Any root $x$ of $\eta^{\prime}(x, n)=0$ is characterized by the condition:

$$
\frac{\tilde{Q}_{3}(x, n)}{\tilde{Q}_{4}(x, n)}-\left(\frac{n-x}{n-1}\right)^{n-1}=0
$$

by Lemma 2.1 and Lemma 2.2. We know that the real analytic function $\widetilde{Q}_{3}(x, n) / \widetilde{Q}_{4}(x, n)-((n-x) /(n-1))^{n-1}$ is negative at $x=0$ and $x=n$ and equals to

$$
\frac{n\left(n^{2}-n-3\right)}{216(n-1)^{3}}(x-1)^{4}+O\left((x-1)^{5}\right) \quad \text { near } x=1 \text {, }
$$

and hence we see that

$$
\frac{d}{d x} \frac{\widetilde{Q}_{3}(x, n)}{\widetilde{Q}_{4}(x, n)}+\left(\frac{n-x}{n-1}\right)^{n-2} \geqq 0 \text { or } \leqq 0
$$

at this root $x$, if it changes the sign from negative to positive there as $\alpha$ or from positive to negative there as $\beta$, respectively. Using (2.5), (2.6) is clearly equivalent to

$$
\begin{aligned}
& (n-x)\left\{\tilde{Q}_{3}^{\prime}(x, n) \tilde{Q}_{4}(x, n)-\tilde{Q}_{3}(x, n) \tilde{Q}_{4}^{\prime}(x, n)\right\} \\
& +(n-1) \tilde{Q}_{3}(x, n) \tilde{Q}_{4}(x, n) \geqq 0 \text { or } \leqq 0 .
\end{aligned}
$$

Next by a little long computation we obtain

$$
\begin{aligned}
& \tilde{Q}_{3}^{\prime}(x, n) \tilde{Q}_{4}(x, n)-\tilde{Q}_{3}(x, n) \tilde{Q}_{4}^{\prime}(x, n) \\
& =\left\{3\left(4 n^{2}+2 n-3\right) x^{2}-16(n-1)\left(n^{2}+5 n-3\right) x+3\left(16 n^{3}-40 n^{2}+24 n-3\right)\right\} \\
& \times\left\{(n-1)(2 n-1)(2 n-3) x^{4}+(2 n-3)\left(2 n^{2}-13 n+8\right) x^{3}\right. \\
& \left.+3\left(8 n^{3}-26 n^{2}+21 n-3\right) x^{2}+n\left(28 n^{2}-52 n+15\right) x+6 n^{2}(2 n-1)\right\} \\
& -\left\{\left(4 n^{2}+2 n-3\right) x^{3}-8(n-1)\left(n^{2}+5 n-3\right) x^{2}\right. \\
& \left.+3\left(16 n^{3}-40 n^{2}+24 n-3\right) x+2 n(4 n-1)(4 n-3)\right\} \\
& \times\left\{4(n-1)(2 n-1)(2 n-3) x^{3}+3(2 n-3)\left(2 n^{2}-13 n+8\right) x^{2}\right. \\
& \left.+6\left(8 n^{3}-26 n^{2}+21 n-3\right) x+n\left(28 n^{2}-52 n+15\right)\right\}
\end{aligned}
$$




$$
\begin{aligned}
=-(n-1)[( & \left.4 n^{2}+2 n-3\right)\left(4 n^{2}-8 n+3\right) x^{6} \\
& -16(n-1)\left(n^{2}+5 n-3\right)\left(4 n^{2}-8 n+3\right) x^{5} \\
& +\left(544 n^{5}-2592 n^{4}+5280 n^{3}-5548 n^{2}+2964 n-630\right) x^{4} \\
+ & 4\left(224 n^{5}-1352 n^{4}+2868 n^{3}-2634 n^{2}+999 n-108\right) x^{3} \\
& +\left(1760 n^{5}-7984 n^{4}+11216 n^{3}-6144 n^{2}+1206 n-81\right) x^{2} \\
+ & 12\left(144 n^{5}-344 n^{4}+272 n^{3}-78 n^{2}+9 n\right) x \\
+ & \left.2\left(160 n^{5}-256 n^{4}+108 n^{3}-18 n^{2}\right)\right]=-(n-1)(\#)
\end{aligned}
$$

where (\#) denotes the polynomial in the brackets. Then, we obtain furthermore

$$
\begin{aligned}
& \frac{n-x}{n-1}\left\{\tilde{Q}_{3}^{\prime}(x, n) \tilde{Q}_{4}(x, n)-\tilde{Q}_{3}(x, n) \tilde{Q}_{4}^{\prime}(x, n)\right\} \\
& +\tilde{Q}_{3}(x, n) \tilde{Q}_{4}(x, n)=(x-n)(\#)+\tilde{Q}_{3}(x, n) \tilde{Q}_{4}(x, n) \\
& =\left(16 n^{4}-24 n^{3}-16 n^{2}+30 n-9\right) x^{7} \\
& -\left(80 n^{5}+104 n^{4}-992 n^{3}+1438 n^{2}-777 n+144\right) x^{6} \\
& +\left(64 n^{6}+672 n^{5}-3568 n^{4}+6688 n^{3}-6316 n^{2}+3108 n-630\right) x^{5} \\
& -\left(544 n^{6}-3488 n^{5}+10688 n^{4}-17020 n^{3}+13500 n^{2}-4626 n+432\right) x^{4} \\
& -\left(896 n^{6}-7168 n^{5}+19456 n^{4}-21752 n^{3}+10140 n^{2}-1638 n+81\right) x^{3} \\
& -\left(1760 n^{6}-9712 n^{5}+15344 n^{4}-9408 n^{3}+2142 n^{2}-189 n\right) x^{2} \\
& -\left(1728 n^{6}-4448 n^{5}+3776 n^{4}-1152 n^{3}+144 n^{2}\right) x \\
& -320 n^{6}+512 n^{5}-216 n^{4}+36 n^{3} \\
& +\left\{\left(4 n^{2}+2 n-3\right) x^{3}-8(n-1)\left(n^{2}+5 n-3\right) x^{2}\right. \\
& \left.+3\left(16 n^{3}-40 n^{2}+24 n-3\right) x+2 n(4 n-1)(4 n-3)\right\} \\
& \times\left\{(n-1)(2 n-1)(2 n-3) x^{4}+(2 n-3)\left(2 n^{2}-13 n+8\right) x^{3}\right. \\
& \left.+3\left(8 n^{3}-26 n^{2}+21 n-3\right) x^{2}+n\left(28 n^{2}-52 n+15\right) x+6 n^{2}(2 n-1)\right\} \\
& =n\left[\left(4 n^{2}+2 n-3\right)\left(4 n^{2}-8 n+3\right) x^{7}-\left(32 n^{5}+96 n^{4}-328 n^{3}+56 n^{2}+240 n-108\right) x^{6}\right. \\
& +\left(224 n^{5}-160 n^{4}-736 n^{3}+636 n^{2}+324 n-270\right) x^{5} \\
& -\left(416 n^{5}-928 n^{4}-216 n^{3}+1232 n^{2}-168 n-252\right) x^{4} \\
& +\left(160 n^{5}-1040 n^{4}+800 n^{3}+680 n^{2}-498 n-81\right) x^{3} \\
& +\left(256 n^{5}+256 n^{4}-752 n^{3}+168 n^{2}+216 n\right) x^{2} \\
& \left.-\left(256 n^{5}-160 n^{4}-120 n^{3}+180 n^{2}\right) x+64 n^{5}-64 n^{4}+48 n^{3}\right] \\
& =n(x-1)^{3} P_{4}(x, n) \text {, }
\end{aligned}
$$

where 


$$
\begin{aligned}
P_{4}(x, n):= & (2 n-1)(2 n-3)\left(4 n^{2}+2 n-3\right) x^{4} \\
& -\left(32 n^{5}+48 n^{4}-256 n^{3}+104 n^{2}+150 n-81\right) x^{3} \\
& +4 n\left(32 n^{4}-88 n^{3}+26 n^{2}+93 n-54\right) x^{2} \\
& +4 n^{2}\left(16 n^{3}+8 n^{2}-66 n+45\right) x-16 n^{3}\left(4 n^{2}-4 n+3\right) .
\end{aligned}
$$

Thus, we obtain a rule for roots $\gamma$ of $\eta^{\prime}(x, n)=0$ as follows, which we denote here by $(R)$ :

Case $0<\gamma<1 . \quad P_{4}(\gamma, n) \leqq 0$, if $\frac{\tilde{Q}_{3}(x, n)}{\widetilde{Q}_{4}(x, n)}-\left(\frac{n-x}{n-1}\right)^{n-1}$ changes its sign from negative to positive at $x=\gamma$ as $\alpha$, otherwise $P_{4}(\gamma, n) \geqq 0$.

Case $1<\gamma<n . \quad P_{4}(\gamma, n) \leqq 0$, if this function changes its sign from positive to negative at $x=\gamma$ as $\beta$, otherwise $P_{4}(\gamma, n) \geqq 0$.

Now, for the above biquadratic polynomial $P_{4}(x, n)$ of $x$ we have the following :

$$
\begin{aligned}
& P_{4}(0, n)=-16 n^{3}\left(4 n^{2}-4 n+3\right)<0 \text { for } n>1, \\
& \begin{aligned}
P_{4}(1, n) & =96 n^{5}-288 n^{4}+24 n^{3}+432 n^{2}-336 n+72 \\
& =24(n-1)(2 n-1)^{2}\left(n^{2}-n-3\right)>0 \text { for } n>\frac{1+\sqrt{13}}{2}, \\
P_{4}(n, n) & =-16 n^{8}+56 n^{7}-48 n^{6}-2 n^{5}+13 n^{4}-3 n^{3} \\
& =-n^{3}(n-1)(2 n-1)(2 n+1)\left(4 n^{2}-10 n+3\right)<0 \text { for } n>\frac{5+\sqrt{13}}{4},
\end{aligned}
\end{aligned}
$$

and $(2 n-1)(2 n-3)\left(4 n^{2}+2 n-3\right)>0$ for $n>3 / 2$, from which we see that $P_{4}(x, n)=0$ has a unique root $\alpha_{0}$ and $\beta_{0}$ in $0<x<1$ and $1<x<n$, respectively, when $n>\frac{1+\sqrt{13}}{2}$. Thus, we see that when $n>\frac{1+\sqrt{13}}{2}=2.3027756 \cdots$, then

$$
\left\{\begin{array}{l}
P_{4}(x, n)<0 \text { for } 0 \leqq x<\alpha_{0} \text { and } \beta_{0}<x \leqq n, \\
P_{4}(x, n)>0 \text { for } \alpha_{0}<x<\beta_{0}, 0<\alpha_{0}<1<\beta_{0}<n,
\end{array}\right.
$$

which implies $0<\alpha \leqq \alpha_{0}$ and $\beta_{0} \leqq \beta<n$. By means of the above mentioned rule (R), we see that $\eta^{\prime}(x, n)=0$ has no solutions in $0<x<\alpha$ and $\beta<x<n$ and $\eta(x)$ is monotone in $\alpha<x<\beta$.

Q.E. D.

We shall show the approximate values of $\alpha=\alpha(n)$ with other related constants with step $1 / 100$ in Table A on the final page. Here, we can show roughly the graph of $y=\eta(x, n)$ with $n>\frac{1+\sqrt{13}}{2}$ as in Fig. 3. Then, we see that $\eta(x, n)$ is decreasing in $0<x<\alpha(n)$ and $\beta(n)<x<n$, and increasing in $\alpha(n)<x<\beta(n)$. Thus, when $n>\frac{1+\sqrt{13}}{2}$, let $z(n)$ be the unique solution of 
in $0<x<n$.

$$
\eta(x, n)=0
$$

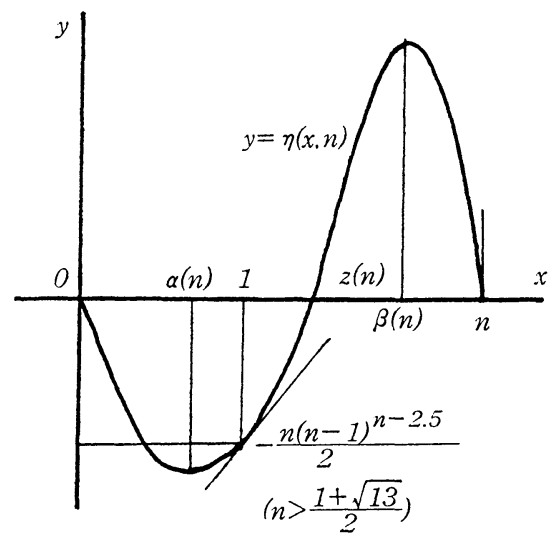

Fig. 3.

Proposition 2. When $\frac{5+\sqrt{13}}{4}<n<\frac{1+\sqrt{13}}{2}, \eta^{\prime}(x, n)=0(0<x<n)$ has two solutions $\alpha$ and $\beta$ such that $1<\alpha<\beta<n$ and $\eta(x, n)$ takes its minimum at $\alpha$ and its maximum at $\beta$, and is monotone in $0<x<\alpha, \alpha<x<\beta$ and $\beta<x<n$, respectively.

Proof. By means of Lemma 2.2, we see easily that $\eta^{\prime}(x, n)=0$ has at least two solutions in $1<x<n$. Let $\alpha$ and $\beta$ be the minimum and the maximum of them. Any root $\gamma$ of $\eta^{\prime}(x, n)=0$ satisfies the rule $(\mathrm{R})$ in the proof of Proposition 1. Since $\tilde{Q}_{3}(x, n) / \widetilde{Q}_{4}(x, n)-((n-x) /(n-1))^{n-1}$ changes its sign from negative to positive at $\alpha$ and from positive to negative at $\beta$, we see that

$$
P_{4}(\alpha, n) \geqq 0 \text { and } P_{4}(\beta, n) \leqq 0 .
$$

Since $P_{4}(0, n)<0$ and $P_{4}(n, n)<0$ and $P_{4}(1)<0$ in this case, we see that $P_{4}(x, n)=0$ has two roots $\alpha_{0}, \beta_{0}$ in $1<x<n$, such that

$$
\begin{array}{ll}
P_{4}(x, n)<0 & \text { for } 0 \leqq x<\alpha_{0} \text { and } \beta_{0}<x \leqq n, \\
P_{4}(x, n) \geqq 0 & \text { for } \alpha_{0} \leqq x \leqq \beta_{0},
\end{array}
$$

and $1<\alpha_{0} \leqq \alpha \leqq \beta_{0} \leqq \beta<n$. On the other hand, if $\eta^{\prime}(x, n)=0$ has roots in $0<x<1$, then at some $\gamma$ of them it must be $P_{4}(\gamma, n) \geqq 0$ by the rule (R), which contradicts to the fact $P_{4}(x, n)<0$ for $0 \leqq x \leqq 1$. Furthermore, if there exist roots other than $\alpha$ and $\beta$ in $1<x<n$, then at some $\gamma_{1}$ and $\gamma_{2}\left(\alpha<\gamma_{1} \leqq \gamma_{2}<\beta\right)$ of them we must have

$$
P_{4}\left(\gamma_{1}, n\right) \leqq 0 \quad \text { and } \quad P_{4}\left(\gamma_{2}, n\right) \geqq 0 \text {, }
$$


from which we have $\gamma_{2} \leqq \beta_{0} \leqq \gamma_{1}$ and hence $\gamma_{1}=\beta_{0}=\gamma_{2}$, which implies that $\eta(x, n)$ is monotone increasing in $\alpha<x<\beta$. We can use the same argument for the intervals $1<x<\alpha$ and $\beta<x<n$.

Q.E.D.

Here, we can show roughly the graph of $y=\eta(x, n)$ with $\frac{5+\sqrt{13}}{4}<n<\frac{1+\sqrt{13}}{2}$ as in Fig. 4. Then, we see that $\eta(x, n)$ is decreasing in $0<x<\alpha(n)$ and $\beta(n)<x<n$ and increasing in $\alpha(n)<x<\beta(n)$. In this case, we can also define $z(n)$ of the unique solution of

in $0<x<n$.

$$
\eta(x, n)=0
$$

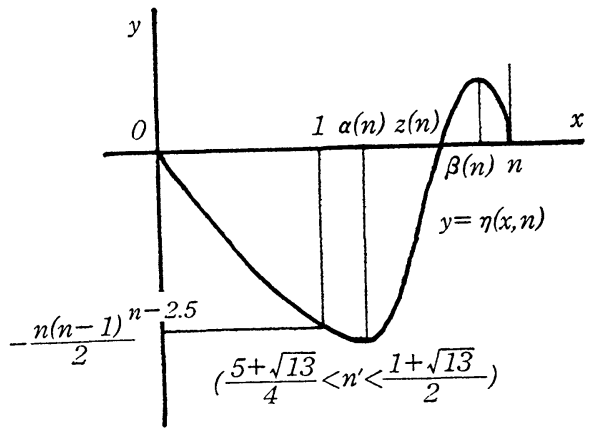

Fig. 4 .

Proposition 3. When $2 \leqq n<\frac{5+\sqrt{13}}{4}, \eta^{\prime}(x, n)$ has a solution $\alpha$ such that $1<\alpha<n$ and $\eta$ takes its minimum at $\alpha$ and is monotone in $0<x<\alpha$ and $\alpha<x<n$, respectively.

Proof. By means of Lemma 2.2, $\eta(x, n)$ takes its minimum in $1 \leqq x \leqq n$ at some points in $1<x<n$. Let $\alpha$ be the minimum of them. By the rule (R), we have $P_{4}(\alpha, n) \geqq 0$. Now, from (2.8) we obtain

$$
P(2 n, n)=-32 n^{3}\left(8 n^{4}-16 n^{3}-2 n^{2}+10 n-3\right)<0,
$$

because

$$
8 n^{4}-16 n^{3}-2 n^{2}+10 n-3=(n-2)\left(8 n^{3}-2 n+6\right)+9 \geqq 9 \text { for } n \geqq 2 \text {. }
$$

Since we have

$$
P_{4}(n, n)>0 \text { for } 2 \leqq n<\frac{5+\sqrt{13}}{4} .
$$

Hence, $P_{4}(x, n)=0$ has roots in $n<x<2 n$ and $2 n<x<+\infty$. Thus, we see that $P_{4}(x, n)=0$ has only one root $\alpha_{0}$ in $0<x<n$ and $1<\alpha_{0}<n$, and 


$$
\begin{array}{ll}
P_{4}(x, n)<0 & \text { for } 0 \leqq x<\alpha_{0}, \\
P_{4}(x, n)>0 & \text { for } \alpha_{0}<x \leqq n,
\end{array}
$$

and $\alpha_{0} \leqq \alpha$. Using the rule $(\mathrm{R})$, we see easily that $\eta^{\prime}(x, n)$ has no solution in $0<x<1$ and $\alpha<x<n$ and $\eta(x, n)$ is monotone decreasing in $0<x<\alpha$ and increasing in $\alpha<x<n$.

Q.E. D.

Here, we can show roughly the graph of $y=\eta(x, n)$ with $2 \leqq n<\frac{5+\sqrt{13}}{4}$ as in Fig. 5.

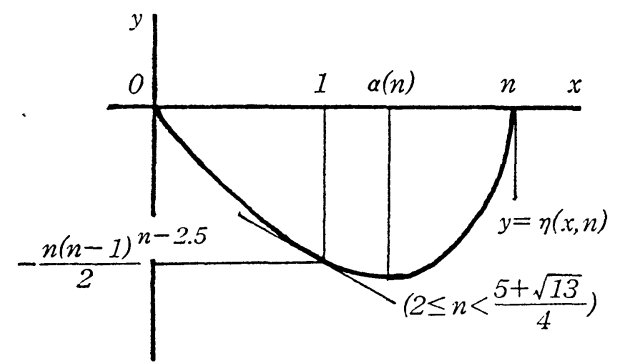

Fig. 5 .

\section{$\S 3$. Some auxiliary constants depending on $n$.}

On the constant $z(n)$ defined at the end of $\S 2$, we obtain by Proposition 1 and Proposition 2 the following

LEMMA 3.1. When $n>\frac{5+\sqrt{13}}{4}$, a constant $b(1<b<n)$ is greater than $z(n)$ if and only if

$$
-\tilde{Q}_{1}(b, n)+\left(\frac{n-b}{n-1}\right)^{n-1} \tilde{Q}_{2}(b, n)>0 .
$$

Now, looking over the data of $z(n)$ computed in $\frac{1+\sqrt{13}}{2}<n \leqq 5$ by a computer (Table A) the following inequality will be assumed

$$
z(n)<1+\frac{7(n-1)}{2(15 n-32)} .
$$

LEMMA 3.2. (3.2) holds for $2.4 \leqq n \leqq 10$.

Proof. For $b=1+\frac{7(n-1)}{2(15 n-32)}=\frac{37 n-71}{2(15 n-32)}$, from (1.14), (1.15) we obtain

$$
\tilde{Q}_{1}(b, n)=\frac{n-1}{2(15 n-32)}\left(332 n^{2}-962 n+405\right),
$$




$$
\tilde{Q}_{2}(b, n)=\frac{n-1}{4(15 n-32)^{2}}\left(12578 n^{3}-59607 n^{2}+83434 n-28755\right)
$$

and

$$
\left(\frac{n-b}{n-1}\right)^{n-1}=\left(\frac{30 n-71}{2(15 n-32)}\right)^{n-1} .
$$

By Lemma 3.1, (3.2) is equivalent to

$$
\left(\frac{30 n-64}{30 n-71}\right)^{n-1}<\frac{12578 n^{3}-59607 n^{2}+83434 n-28755}{2(15 n-32)\left(332 n^{2}-962 n+405\right)} .
$$

Now, we first show that

$$
\left(\frac{30 n-64}{30 n-71}\right)^{n-1} \searrow \text { in } \frac{71}{30}=2.3 \dot{6}<n<+\infty .
$$

In fact, its logarithmic derivative is

$$
\log \frac{30 n-64}{30 n-71}+\frac{34}{30 n-64}-\frac{41}{30 n-71} \longrightarrow 0 \quad(\text { as } n \rightarrow+\infty) .
$$

Furthermore, the derivative of this function of $n$ is

$$
\frac{30(15750 n-35266)}{(30 n-64)^{2}(30 n-71)^{2}},
$$

which is positive for $n>\frac{71}{30}\left(>\frac{35266}{15750}=\frac{2519}{1125}=2.239 i\right)$.

Hence we obtain

$$
\frac{d}{d n}\left(\frac{30 n-64}{30 n-71}\right)^{n-1}<0 \text { for } n>\frac{71}{30} .
$$

Then, we show that the right hand side of (3.3) is also decreasing in $2.18<$ $n<+\infty$. In fact, we have

$$
\begin{aligned}
& \frac{d}{d n}\left(\frac{12578 n^{3}-59607 n^{2}+83434 n-28755}{2(15 n-32)\left(332 n^{2}-962 n+405\right)}\right) \\
& =-\left(18286352 n^{4}-96222364 n^{3}+166131917 n^{2}-104157900 n\right. \\
& \quad+21424095) /\left\{2(15 n-32)^{2}\left(332 n^{2}-962 n+405\right)^{2}\right\},
\end{aligned}
$$

which is negative for $n \geqq 2.18$, because we see easily that

$$
18286352 n^{4}-96222364 n^{3}+166131917 n^{2}-104157900 n+21424095
$$

is increasing in $2.13<n<+\infty$ and $=2031.8772$ at $n=2.18$.

On the other hand

$$
\lim _{n \rightarrow+\infty}\left(\frac{30 n-64}{30 n-71}\right)^{n-1}=e^{7 / 30}=1.262802343 \cdots
$$


and

$$
\lim _{n \rightarrow+\infty} \frac{12578 n^{3}-59607 n^{2}+83434 n-28755}{2(15 n-32)\left(332 n^{2}-962 n+405\right)}=\frac{12578}{2 \times 15 \times 332}=1.262851406 \cdots .
$$

Denoting the left hand side and the right one of (3.3) by $L(n)$ and $R(n)$, respectively, and computing their values with steps $1 / 1000$ and $1 / 10000$ in the following intervals, we see that

and

$$
L(n)<R(n+0.001) \quad \text { in } 2.400 \leqq n \leqq 2.580
$$

$$
L(n)<R(n+0.0001) \quad \text { in } 2.5800 \leqq n \leqq 10.0000 \text {, }
$$

which implies

$$
L(n)<R(n) \text { for } 2.4 \leqq n \leqq 10
$$

by means of the decreasing property of $L(n)$ and $R(n)$.

Q.E.D.

We have the following proposition which is important for the purpose of this work in order to use the same method as the one in the proof of Lemma 3.2 and will be proved in another paper [22].

Proposition 4. $z(n)$ is decreasing in $\frac{5+\sqrt{13}}{4}+\frac{11}{1000}<n<22$, and hence

$$
z(n)<\frac{5+\sqrt{13}}{4}+\frac{11}{1000} \text { for } \frac{5+\sqrt{13}}{4}+\frac{11}{1000} \leqq n \leqq 22 .
$$

We have more sharp evaluations as follows:

i ) $z(n)<2.1$ for $2.250 \leqq n \leqq 2.400$,

ii) $z(n)<2$ for $2.300 \leqq n \leqq 2.550$,

iii) $z(n)<1.85$ for $2.50 \leqq n \leqq 3.00$,

iv) $z(n)<1.65$ for $2.80 \leqq n \leqq 3.80$,

v) $z(n)<1.38$ for $3.60 \leqq n \leqq 6.10$,

vi) $z(n)<1.1$ for $6.0 \leqq n \leqq 22$.

Proposition 5. $\quad w(n):=X_{n}^{-1}(z(n))$ is increasing in $\frac{5+\sqrt{13}}{4}+\frac{11}{1000}<n<22$.

Proof. For $z=z(n)$ and $w=w(n)$, we have $w(n-w)^{n-1}=z(n-z)^{n-1}$, from which we obtain by differentiation with respect to $n$

$$
\frac{n(1-w)}{w(n-w)} w^{\prime}=-\frac{n(z-1)}{z(n-z)} z^{\prime}+(n-1)\left(\frac{1}{n-z}-\frac{1}{n-w}\right)+\log \frac{n-z}{n-w} .
$$

By means of Proposition 4, we see that

$$
-\frac{n(z-1)}{z(n-z)} z^{\prime}>0 \quad \text { for } \frac{5+\sqrt{13}}{4}+\frac{11}{1000} \leqq n \leqq 22 \text {. }
$$


Setting $T=(1-w) /(n-1)$ and $S=(z-1) /(n-1)$, we obtain

$$
\begin{aligned}
&(n-1)\left(\frac{1}{n-z}-\frac{1}{n-w}\right)+\log \frac{n-z}{n-w} \\
&=\frac{1}{1-S}-\frac{1}{1+T}+\log \frac{1-S}{1+T} \\
&=\left(1+\frac{1}{2} S^{2}+\frac{2}{3} S^{3}+\cdots+\frac{m-1}{m} S^{m}+\cdots\right) \\
&-\left(1+\frac{1}{2} T^{2}-\frac{2}{3} T^{3}+\cdots+(-1)^{m} \frac{m-1}{m} T^{m}+\cdots\right) \\
&= \frac{1}{2}\left(S^{2}-T^{2}\right)+\frac{2}{3}\left(S^{3}+T^{3}\right)+\frac{3}{4}\left(S^{4}-T^{4}\right)+\cdots \\
&+\frac{2 m-1}{2 m}\left(S^{2 m}-T^{2 m}\right)+\frac{2 m}{2 m+1}\left(S^{2 m+1}+T^{2 m+1}\right)+\cdots>0,
\end{aligned}
$$

since we have $S>T$ by Proposition 4 in (IV) for $n>2$.

Q.E.D.

Remark. The monotony of $z(n)$ and $w(n)$ with respect to $n$ was proved for $\frac{5+\sqrt{13}}{4}+\frac{11}{1000}<n<22$, and the right end 22 may be replaced by $+\infty$, but the left end cannot be replaced by $\frac{5+\sqrt{13}}{4}+\frac{1}{100}$, which will be shown in [22].

As started in $\S 1$, we wish to prove the inequality

$$
\eta\left(X_{n}(x), n\right)-\eta(x, n)>0
$$

for $0<x<1$ and $n>\frac{5+\sqrt{13}}{4}+\frac{11}{1000}$. By means of Proposition 1 and Proposition 2 , we see easily that (3.5) holds for

$$
0 \leqq x \leqq w(n) \text { and } n>\frac{5+\sqrt{13}}{4} \text {. }
$$

LEMMA 3.3. If $\alpha(n) \leqq w(n)$ for $n>\frac{1+\sqrt{13}}{2}$, then (3.5) holds for $0<x<1$.

Proof. By means of Proposition $1, \eta(x, n)$ is increasing in $\alpha(n)<x<z(n)$, hence we have for $w(n) \leqq x<1$

$$
\eta\left(X_{n}(x), n\right)>\eta(x, n)
$$

because $\alpha(n) \leqq w(n) \leqq x<X_{n}(x) \leqq X_{n}(w(n))=z(n)$. Therefore, combining this with the fact mentioned above, we obtain this lemma.

Q.E. D.

On the other hand, by means of an analogous way in the proof of Proposition 4 we can prove the following proposition ([22]). 
Proposition 6. $\alpha(n)$ and $\beta(n)$ in Proposition 1 are decreasing and increasing in $\frac{1+\sqrt{13}}{2}<n<22$, respectively.

The right end 22 may be replaced by $+\infty$. Now, looking Table A we find as follows:

$$
\begin{array}{ll}
\alpha(3.23)=0.644034 \cdots, & w(3.23)=0.643027 \cdots, \\
\alpha(3.24)=0.642357 \cdots, & w(3.24)=0.645647 \cdots, \\
\alpha(3.25)=0.640700 \cdots, & w(3.25)=0.648237 \cdots .
\end{array}
$$

Hence, we obtain from Propositions 5 and 6 and Lemma 3.3 the following

LEMMA 3.4. When $3.24 \leqq n \leqq 22$, (3.5) holds for $0<x<1$.

In the following, by virtue of Lemma 3.4 we shall treat mainly values of $n$ such

and

$$
w(n)<\alpha(n)
$$

$$
\frac{1+\sqrt{13}}{2}<n<3.24
$$

For such $n$ we define $x=Z(n)$ by

and

$$
\alpha(n)<Z(n)<z(n)
$$

$$
\eta(w(n), n)=\eta(Z(n), n) .
$$

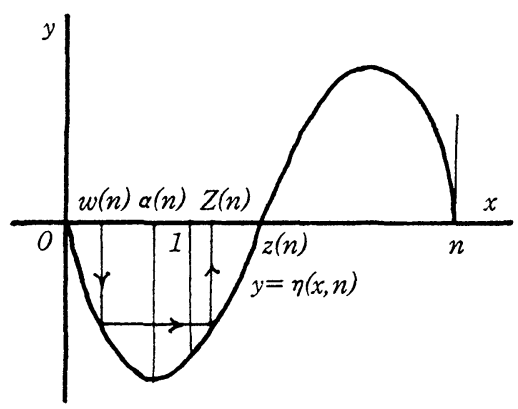

Fig. 6.

LEMMA 3.5. If $w(n)<\alpha(n)$ and $Z(n) \leqq 1$, then (3.5) holds for $0<x<1$.

Proof. By means of Proposition 1, for $w(n)<x<1$ we have

$$
\eta(x, n)<\eta(1, n)<\eta\left(X_{n}(x), n\right) .
$$


Hence, we obtain this lemma.

Q.E.D.

Now, we consider the case: $w(n)<\alpha(n)$ and $1<Z(n)(<z(n))$. We wish to show that $Z(n)$ is decreasing with respect to $n$. Differentiating (3.6) with respect to $n$ we obtain

$$
\frac{\partial \eta}{\partial x}(Z, n) Z^{\prime}=\frac{\partial \eta}{\partial x}(w, n) w^{\prime}+\frac{\partial \eta}{\partial n}(w, n)-\frac{\partial \eta}{\partial n}(Z, n),
$$

where $w=w(n)$ and $Z=Z(n)$.

Since we have $\frac{\partial \eta}{\partial x}(w, n)<0, \frac{\partial \eta}{\partial x}(Z, n) \geqq 0$ and $w^{\prime}>0$ for $n>\frac{5+\sqrt{13}}{4}+\frac{11}{1000}$ by Propositions 1,2 and 5 , we can claim $Z^{\prime}<0$ if we have

$$
\frac{\partial \eta}{\partial n}(w, n) \leqq \frac{\partial \eta}{\partial n}(Z, n) \text {. }
$$

Since we have from (1.16)

$$
\begin{aligned}
\frac{\partial \eta}{\partial n}(x, n)= & \frac{x}{(x-1)^{3}}(n-1)^{n-2} \sqrt{n-x} \\
& \times\left\{\left(\frac{d}{d n} \log (n-1)^{n-2}+\frac{1}{2(n-x)}\right) \times\left(-\tilde{Q}_{1}+\left(\frac{n-x}{n-1}\right)^{n-1} \tilde{Q}_{2}\right)\right. \\
& \left.\quad+\frac{\partial}{\partial n}\left(-\tilde{Q}_{1}+\left(\frac{n-x}{n-1}\right)^{n-1} \tilde{Q}_{2}\right)\right\} \\
= & \eta(x, n) \frac{d}{d n} \log (n-1)^{n-2}+\frac{x}{2(x-1)^{3} \sqrt{n-x}} \Lambda(x, n),
\end{aligned}
$$

where we set

$$
\begin{aligned}
\Lambda(x, n):= & -\tilde{Q}_{1}(x, n)+\left(\frac{n-x}{n-1}\right)^{n-1} \tilde{Q}_{2}(x, n) \\
& +2(n-x) \frac{\partial}{\partial n}\left\{-\tilde{Q}_{1}(x, n)+\left(\frac{n-x}{n-1}\right)^{n-1} \tilde{Q}_{2}(x, n)\right\},
\end{aligned}
$$

using (3.6), (3.7) is equivalent to

$$
\frac{w \Lambda(w, n)}{(w-1)^{3} \sqrt{n-w}} \leqq \frac{Z \Lambda(Z, n)}{(Z-1)^{3} \sqrt{n-Z}} .
$$

Now, by (1.14) and (1.15) we obtain

$$
\begin{aligned}
\Lambda(x, n)= & -\left\{\tilde{Q}_{1}(x, n)+2(n-x) \frac{\partial}{\partial n} \tilde{Q}_{1}(x, n)\right\} \\
+ & \left(\frac{n-x}{n-1}\right)^{n-1}\left\{\tilde{Q}_{2}(x, n)+2(n-x) \frac{\partial}{\partial n} \tilde{Q}_{2}(x, n)\right. \\
& \left.+2(n-x) \tilde{Q}_{2}(x, n)\left(\log \frac{n-x}{n-1}+\frac{x-1}{n-x}\right)\right\}
\end{aligned}
$$




$$
\begin{aligned}
&=-\left\{4(4 n+1) x^{2}-5\left(4 n^{2}+14 n-7\right) x+80 n^{2}-48 n+3\right\} \\
&+\left(\frac{n-x}{n-1}\right)^{n-1}\left\{-2(4 n-5) x^{3}+\left(10 n^{2}-31 n+23\right) x^{2}\right. \\
&+\left(20 n^{2}-54 n+9\right) x+3 n(10 n-3)+2(n-x) \tilde{Q}_{2}(x, n) \\
&\left.\quad \times\left(\log \frac{n-x}{n-1}+\frac{x-1}{n-x}\right)\right\} .
\end{aligned}
$$

LEMMA 3.6. Regarding $\Lambda(x, n)$ we have the following:

i) $\Lambda(0, n)>0$ for $n \geqq 2$,

ii) $\Lambda(1+(n-1) t, n)=-\frac{(n-1)^{2}(n-2)}{2} t^{3}+\frac{n(n-1)^{2}\left(10 n^{3}-25 n^{2}+19 n+2\right)}{12} t^{4}$ $+\cdots$ near $x=1$,

iii) $\lim _{x \rightarrow n-0} \Lambda(x, n)=(n-1)\left(4 n^{2}-10 n+3\right)$.

Proof. i) From (3.10) and (1.15), we obtain

$$
\begin{aligned}
\Lambda(0, n)= & -\left(80 n^{2}-48 n+3\right)+\left(\frac{n}{n-1}\right)^{n-1} \\
& \times 3 n\left\{10 n-3+2 n(2 n-1)\left(\log \frac{n}{n-1}-\frac{1}{n}\right)\right\} .
\end{aligned}
$$

Since we have

$$
\log \frac{n}{n-1}-\frac{1}{n}=\frac{1}{2 n^{2}}+\frac{1}{3 n^{3}}+\cdots>\frac{3 n+2}{6 n^{3}}
$$

$\Lambda(0, n)>0$ is implied from the inequality:

$$
-\left(80 n^{2}-48 n+3\right)+\left(\frac{n}{n-1}\right)^{n-1} 3 n\left\{10 n-3+\frac{(2 n-1)(3 n+2)}{3 n^{2}}\right\}>0
$$

i.e.

$$
\left(\frac{n}{n-1}\right)^{n-1}>\frac{n\left(80 n^{2}-48 n+3\right)}{30 n^{3}-3 n^{2}+n-2}:=h(n)
$$

We shall prove that (3.11) holds for $n \geqq 2$. First of all, we see that

$$
\left[\left(\frac{n}{n-1}\right)^{n-1}\right]_{n=2}=2>h(2)=\frac{2 \times 227}{228}
$$

and

$$
\lim _{n \rightarrow+\infty}\left(\frac{n}{n-1}\right)^{n-1}=e=2.71828 \cdots>\lim _{n \rightarrow+\infty} h(n)=\frac{8}{3} .
$$

If (3.11) does not hold for $2<n<+\infty$, there exist $2<n_{1} \leqq n_{2}<+\infty$ such that

$$
f\left(n_{1}\right) \leqq g\left(n_{1}\right) \text { and } f\left(n_{2}\right) \geqq g\left(n_{2}\right),
$$


where

$$
\begin{aligned}
& f(n)=\frac{d}{d n} \log \left(\frac{n}{n-1}\right)^{n-1}=\log \frac{n}{n-1}-\frac{1}{n}, \\
& g(n)=\frac{d}{d n} \log h(n)=\frac{1200 n^{4}-20 n^{3}-519 n^{2}+192 n-6}{n\left(80 n^{2}-48 n+3\right)\left(30 n^{3}-3 n^{2}+n-2\right)} .
\end{aligned}
$$

We shall show that $f(n)>g(n)$ for $2 \leqq n \leqq 34.9$. $f(n)>g(n)$ is implied from

$$
\frac{1}{2 n^{2}}+\frac{1}{3 n^{3}}+\frac{1}{4 n^{4}} \geqq g(n),
$$

which is equivalent to

$$
\frac{4 n+3}{12 n^{2}} \geqq \frac{1640 n^{4}-1352 n^{3}+601 n^{2}-111 n+6}{2\left(80 n^{2}-48 n+3\right)\left(30 n^{3}-3 n^{2}+n-2\right)},
$$

and hence

$$
-240 n^{6}+8592 n^{5}-7390 n^{4}+740 n^{3}-291 n^{2}+273 n-18 \geqq 0 .
$$

The left hand side of the above inequality

$$
\geqq 148.4 n^{4}+740 n^{3}-291 n^{2}+273 n-18>0 \text { for } 2 \leqq n \leqq 34.9 \text {, }
$$

since we have $8592 / 2 \times 240=17.9$.

Next, we shall show that $f(n)<g(n)$ for $36 \leqq n<+\infty . \quad f(n)<g(n)$ is implied from

$$
\frac{1}{2 n^{2}}+\frac{1}{3 n^{3}}+\frac{1}{4 n^{3}(n-1)} \leqq g(n),
$$

which is equivalent to

$$
\frac{4 n-1}{12 n(n-1)} \leqq \frac{1640 n^{4}-1352 n^{3}+601 n^{2}-111 n+6}{2\left(80 n^{2}-48 n+3\right)\left(30 n^{3}-3 n^{2}+n-2\right)},
$$

and hence

$$
-240 n^{6}+8832 n^{5}-8782 n^{4}+3090 n^{3}-89 n^{2}-87 n+6 \leqq 0
$$

The left hand side of the above inequality

$$
\begin{aligned}
& \leqq-1870 n^{4}+3090 n^{3}-89 n^{2}-87 n+6 \\
& \leqq-2312369 n^{2}-87 n+6<0 \text { for } 36 \leqq n<+\infty
\end{aligned}
$$

Thus we see that $34.9<n_{1} \leqq n_{2}<36$.

On the other hand, we have

$$
h^{\prime}(n)=\frac{1200 n^{4}-20 n^{3}-519 n^{2}+192 n-6}{\left(30 n^{3}-3 n^{2}+n-2\right)^{2}}>0 \text { for } n \geqq 2
$$

and hence for $33 \leqq n \leqq 36$ we have 


$$
\begin{aligned}
\left(\frac{n}{n-1}\right)^{n-1} & \geqq\left(\frac{33}{32}\right)^{32}=2.676990 \cdots \\
>h(36) & =2.629539 \cdots \geqq h(n),
\end{aligned}
$$

which contradicts to the existence of $n_{1}$ and $n_{2}$.

ii) On the expressions in (3.10), setting $x=1+(n-1) t$, we have

$$
\begin{aligned}
& 4(4 n+1) x^{2}-5\left(4 n^{2}+14 n-7\right) x+80 n^{2}-48 n+3 \\
& =(n-1)\left\{4(4 n+1)(n-1) t^{2}-\left(20 n^{2}+38 n-43\right) t+6(10 n-7)\right\}, \\
& -2(4 n-5) x^{3}+\left(10 n^{2}-31 n+23\right) x^{2}+\left(20 n^{2}-54 n+9\right) x+3 n(10 n-3) \\
& =(n-1)\left\{-2(4 n-5)(n-1)^{2} t^{3}+\left(10 n^{2}-55 n+53\right)(n-1) t^{2}\right. \\
& \left.+\left(40 n^{2}-140 n+85\right) t+6(10 n-7)\right\}, \\
& \tilde{Q}_{2}(x, n)=(n-1)\left\{(2 n-3)(n-1)^{2} t^{2}+\left(8 n^{2}-20 n+9\right) t+6(2 n-1)\right\} \text {, } \\
& \left(\frac{n-x}{n-1}\right)^{n-1}=(1-t)^{n-1} \\
& =1-(n-1) t+\frac{(n-1)(n-2)}{2} t^{2}-\frac{(n-1)(n-2)(n-3)}{6} t^{3} \\
& +\frac{(n-1)(n-2)(n-3)(n-4)}{24} t^{4}+\cdots, \\
& (n-x)\left(\log \frac{n-x}{n-1}+\frac{x-1}{n-x}\right) \\
& =(n-1)\{(1-t) \log (1-t)+t\} \\
& =(n-1)\left\{\frac{1}{2} t^{2}+\frac{1}{2 \cdot 3} t^{3}+\frac{1}{3 \cdot 4} t^{4}+\frac{1}{4 \cdot 5} t^{5}+\cdots\right\} \text {, }
\end{aligned}
$$

from which we obtain

$$
\begin{aligned}
& \Lambda(1+(n-1) t, n)=-\frac{(n-1)^{2}(n-2)}{2} t^{3} \\
& \quad+\frac{n(n-1)^{2}\left(10 n^{3}-25 n^{2}+19 n+2\right)}{12} t^{4}+\cdots \text { near } x=1 .
\end{aligned}
$$

iii) Since we have

$$
\begin{aligned}
{\left[4(4 n+1) x^{2}\right.} & \left.-5\left(4 n^{2}+14 n-7\right) x+80 n^{2}-48 n+3\right]_{x=n} \\
& =-4 n^{3}+14 n^{2}-13 n+3=-(n-1)\left(4 n^{2}-10 n+3\right),
\end{aligned}
$$

and 


$$
\begin{aligned}
\lim _{x \rightarrow n-0}(n-x)\left(\log \frac{n-x}{n-1}+\frac{x-1}{n-x}\right) \\
\quad=(n-1)\left\{\frac{1}{2}+\frac{1}{2 \cdot 3}+\frac{1}{3 \cdot 4}+\frac{1}{4 \cdot 5}+\cdots\right\}=n-1 .
\end{aligned}
$$

We obtain easily

$$
\lim _{x \rightarrow 0} \Lambda(x, n)=(n-1)\left(4 n^{2}-10 n+3\right) .
$$

Q.E.D.

Remark. From Lemma 3.6, we see that when $n>2, \Lambda(x, n)$ is negative for $x>1$ sufficiently near 1 and when $n>\frac{5+\sqrt{13}}{4}, \Lambda(x, n)$ is positive for $x<n$ sufficiently near $n$. For $0<x<1$, we have the following proposition which will be proved also in [22].

Proposition 7. When $n \geqq 2$, we have

$$
\Lambda(x, n)>0 \text { for } 0 \leqq x<1 .
$$

Now, considering (3.9) and Lemma 3.6, we define an auxiliary real analytic function $\Gamma(x, n)$ by

$$
\Gamma(x, n):= \begin{cases}\frac{x \Lambda(x, n)}{(x-1)^{3} \sqrt{n-x}} & \text { for } 0 \leqq x<n, x \neq 1, \\ -\frac{n-2}{(n-1) \sqrt{n-1}} & \text { for } x=1 .\end{cases}
$$

LEMMA 3.7. We have

$$
\left[\frac{\partial}{\partial x} \Gamma(x, n)\right]_{x=1}=\frac{10 n^{4}-25 n^{3}+13 n^{2}+17 n-6}{12(n-1)^{2} \sqrt{n-1}}>0 \text { for } n \geqq 2 \text {. }
$$

Proof. Setting $x=1+(n-1) t$, we obtain from (3.12) and (3.14)

$$
\begin{aligned}
\Gamma(X, n)= & \frac{(1+(n-1) t)}{(n-1)^{3} \sqrt{n-1} \sqrt{1-t}} \\
& \left\{-\frac{(n-1)^{2}(n-2)}{2}+\frac{n(n-1)^{2}\left(10 n^{3}-25 n^{2}+19 n+2\right)}{12} t+\cdots\right\},
\end{aligned}
$$

from which we obtain

$$
\begin{gathered}
{\left[\frac{\partial}{\partial x} \Gamma(x, n)\right]_{x=1}=\frac{d t}{d x} \frac{1}{(n-1)^{3} \sqrt{n-1}}\left[\left\{(n-1)+\frac{1}{2}\right\}\left\{-\frac{(n-1)^{2}(n-2)}{2}\right\}\right.} \\
\left.+\frac{(n-1)^{2}\left(10 n^{3}-25 n^{2}+19 n+2\right)}{12}\right]
\end{gathered}
$$




$$
=\frac{10 n^{4}-25 n^{3}+13 n^{2}+17 n-6}{12(n-1)^{2} \sqrt{n-1}} .
$$

Next, we have

$$
10 n^{4}-25 n^{3}+13 n^{2}+17 n-6=(n-2)\left(10 n^{3}-5 n^{2}+3 n+23\right)+40 \geqq 40
$$

for $n \geqq 2$.

Q.E.D.

In the following, we want to show that $\Gamma(x, n)$ is monotone increasing in $w(n)<x<Z(n)$ with $\frac{5+\sqrt{13}}{4}+\frac{11}{1000} \leqq n \leqq 3.24$.

Differentiating $\Gamma(x, n)$ with respect to $x$, we obtain from (3.10)

$$
\frac{d}{d x}\left\{\frac{x \Lambda(x, n)}{(x-1)^{3} \sqrt{n-x}}\right\}=\frac{1}{2(x-1)^{4}(n-x) \sqrt{n-x}} \cdot \Pi(x, n),
$$

where

$$
\begin{aligned}
& \Pi(x, n)=2 x(x-1)(n-x) \frac{\partial}{\partial x} \Lambda(x, n)+\left\{5 x^{2}-(4 n-1) x-2 n\right\} \Lambda(x, n) \\
& =2 x(x-1)(n-x)\left\langle-8(4 n+1) x+5\left(4 n^{2}+14 n-7\right)\right. \\
& +\left(\frac{n-x}{n-1}\right)^{n-1}\left[-6(4 n-5) x^{2}+2\left(10 n^{2}-31 n+23\right) x+20 n^{2}-54 n+9\right. \\
& +2\left\{2\left(2 n^{2}-5 n+3\right) x+4 n^{2}-10 n+3\right\}(n-x)\left(\log \frac{n-x}{n-1}+\frac{x-1}{n-x}\right) \\
& \left.-2\left\{2\left(2 n^{2}-5 n+3\right) x^{2}+\left(4 n^{2}-10 n+3\right) x+6 n^{2}-3 n\right\} \log \frac{n-x}{n-1}\right] \\
& -\left(\frac{n-x}{n-1}\right)^{n-2}\left[-2(4 n-5) x^{3}+\left(10 n^{2}-31 n+23\right) x^{2}+\left(20 n^{2}-54 n+9\right) x\right. \\
& +30 n^{2}-9 n+2\left\{\left(2 n^{2}-5 n+3\right) x^{2}+\left(4 n^{2}-10 n+3\right) x+6 n^{2}-3 n\right\}(n-x) \\
& \left.\left.\quad \times\left(\log \frac{n-x}{n-1}+\frac{x-1}{n-x}\right)\right]\right\rangle+\left\{5 x^{2}-(4 n-1) x-2 n\right\} \\
& \quad \times\left\langle-4(4 n+1) x^{2}+5\left(4 n^{2}+14 n-7\right) x-\left(80 n^{2}-48 n+3\right)+\left(\frac{n-x}{n-1}\right)^{n-1}\right. \\
& \quad \times\left[-2(4 n-5) x^{3}+\left(10 n^{2}-31 n+23\right) x^{2}\right. \\
& +\left(20 n^{2}-54 n+9\right) x+3 n(10 n-3)+2(n-x)\left\{\left(2 n^{2}-5 n+3\right) x^{2}\right. \\
& \left.\left.\left.+\left(4 n^{2}-10 n+3\right) x+6 n^{2}-3 n\right\}\left(\log \frac{n-x}{n-1}+\frac{x-1}{n-x}\right)\right]\right\rangle,
\end{aligned}
$$

which can be arranged by a long computation as follows:

$$
\Pi(x, n)=A_{4}(x, n)+\left(\frac{n-x}{n-1}\right)^{n-1} \times
$$




$$
\left\{B_{5}(x, n)-2 B_{4}(x, n)(n-x)\left(\log \frac{n-x}{n-1}+\frac{x-1}{n-x}\right)\right\},
$$

$$
\begin{aligned}
A_{4}(x, n):= & -4(4 n+1) x^{4}+5\left(12 n^{2}+26 n-25\right) x^{3} \\
& -8\left(5 n^{3}+48 n^{2}-68 n+15\right) x^{2}+\left(240 n^{3}-552 n^{2}+200 n-3\right) x \\
& +2 n\left(80 n^{2}-48 n+3\right), \\
B_{4}(x, n):= & (n-1)(2 n-1)(2 n-3) x^{4}+\left(4 n^{3}-32 n^{2}+55 n-24\right) x^{3} \\
+3 & \left(8 n^{3}-26 n^{2}+21 n-3\right) x^{2}+n\left(28 n^{2}-52 n+15\right) x+6 n^{2}(2 n-1), \\
B_{5}(x, n):= & 2\left(12 n^{2}-24 n+11\right) x^{5}-\left(20 n^{3}-60 n^{2}+139 n-107\right) x^{4} \\
& -\left(20 n^{3}-240 n^{2}+367 n-102\right) x^{3}-\left(120 n^{3}-402 n^{2}+271 n-21\right) x^{2} \\
& -n\left(140 n^{2}-228 n+39\right) x-6 n^{2}(10 n-3) .
\end{aligned}
$$

LEMMA 3.8. On $A_{4}(x, n)$ we have the following:

i) When $\frac{5+\sqrt{13}}{4}<n \leqq 2.84, A_{4}(x, n)>0$ for $0 \leqq x \leqq n$ and convex upward in $0<x<1$ and decreasing in $1<x<n$.

ii) When $2.84<n \leqq 5, A_{4}(x, n)>0$ for $0 \leqq x \leqq 1.6$ and convex upward in $0<x<1$ and decreasing in $1<x<1.6$.

Proof. From (3.16) we have

$$
\frac{1}{2} A_{4}^{\prime \prime}(x, n)=-24(4 n+1) x^{2}+15\left(12 n^{2}+26 n-25\right) x-8\left(5 n^{3}+48 n^{2}-68 n+15\right) \text {. }
$$

We see easily that

$$
\frac{15\left(12 n^{2}+26 n-25\right)}{2 \times 24(4 n+1)}>\frac{5}{2} \quad \text { for } n \geqq 2 \text {. }
$$

We have

$$
A_{4}^{\prime \prime}(1, n)=2\left(-40 n^{3}-204 n^{2}+838 n-519\right)<0 \text { for } n \geqq 2.05,
$$

hence

$$
A_{4}^{\prime \prime}(x, n)<0 \text { for } 0 \leqq x \leqq 1 \text { with } n \geqq 2.05
$$

and so $A_{4}(x, n)$ is convex upward in $0<x<1$ with $n \geqq 2.05$. We have

$$
A_{4}{ }^{\prime \prime}(n, n)=2\left(44 n^{3}-18 n^{2}+169 n-120\right)>0 \text { for } n \geqq 2 \text {. }
$$

Next, we have

We see easily

$$
\begin{aligned}
A_{4}^{\prime}(x, n)= & -16(4 n+1) x^{3}+15\left(12 n^{2}+26 n-25\right) x^{2} \\
& -16\left(5 n^{3}+48 n^{2}-68 n+15\right) x+240 n^{3}-552 n+200 n-3 .
\end{aligned}
$$




$$
\begin{aligned}
& A_{4}^{\prime}(0, n)=240 n^{3}-552 n^{2}+200 n-3 \geqq 56 n-3 \geqq 109 \text { for } n \geqq 2, \\
& A_{4}^{\prime}(1, n)=160 n^{3}-1140 n^{2}+1614 n-634<0 \text { for } 2 \leqq n<5.3, \\
& A_{4}^{\prime}(n, n)=36 n^{4}-154 n^{3}+161 n^{2}-40 n-3<0 \\
& \quad \text { for } 2 \leqq n \leqq 2.848 \text { and }>0 \text { for } 2.849 \leqq n<+\infty
\end{aligned}
$$

and

$$
A_{4}^{\prime}(1.6, n)=112 n^{3}-1320 n^{2}+2677.056 n-1412.536<0 \text { for } 2 \leqq n \leqq 9 .
$$

Last, we have

$$
\begin{aligned}
A_{4}(0, n)=2 n\left(80 n^{2}-48 n+3\right)>0 & \text { for } n \geqq 2, \\
A_{4}(1, n)=36(n-1)^{2}(10 n-7)>0 & \text { for } n \geqq 2, \\
A_{4}(n, n)=n(n-1)^{2}\left(4 n^{2}-10 n+3\right)>0 & \text { for } n>\frac{5+\sqrt{13}}{4}
\end{aligned}
$$

and

$$
A_{4}(1.6, n)=441.6 n^{3}-1716.48 n^{2}+2146.2624 n-850.2144>0 \text { for } n \geqq 2 .
$$

Taking account of these facts, we obtain the claim of this lemma. Q.E.D.

Remark. The reason of noticing the value 1.6 is

$$
z(n) \leqq z(2.84)=1.579536 \cdots \text { for } n \geqq 2.84
$$

and $z(2.8)=1.602411 \cdots($ see Table A $)$.

LEMMA 3.9. When $n \geqq 2, B_{4}(x, n)$ is positive for $0 \leqq x \leqq n$ and increasing in $0<x<n$.

Proof. We have from (3.17)

$$
\begin{aligned}
B_{4}(x, n)= & (n-1)(2 n-1)(2 n-3) x^{4}+(2 n-3)\left(2 n^{2}-13 n+8\right) x^{3} \\
& +3(n-1)\left(8 n^{2}-18 n+3\right) x^{2}+n(2 n-3)(14 n-5) x+6 n^{2}(2 n-1), \\
B_{4}^{\prime}(x, n)= & 4 n(n-1)(2 n-1)(2 n-3) x^{3}+3(2 n-3)\left(2 n^{2}-13 n+8\right) x^{2} \\
& +6(n-1)\left(8 n^{2}-18 n+3\right) x+n(2 n-3)(14 n-5), \\
\frac{1}{6} B_{4}^{\prime \prime}(x, n)= & 2(n-1)(2 n-1)(2 n-3) x^{2} \\
& +(2 n-3)\left(2 n^{2}-13 n+8\right) x+(n-1)\left(8 n^{2}-18 n+3\right),
\end{aligned}
$$

from which we see

$$
\begin{aligned}
& B_{4}(0, n)=6 n^{2}(2 n-1)>0 \text { for } n>0.5 \\
& B_{4}^{\prime}(0, n)=n(2 n-3)(14 n-5)>0 \text { for } n>1.5 .
\end{aligned}
$$


The discriminant $D$ of $B_{4}^{\prime \prime}(x, n) / 6$ as a quadratic polynomial of $x$ is given by

$$
\begin{aligned}
D & =(2 n-3)^{2}\left(2 n^{2}-13 n+8\right)^{2}-8(n-1)^{2}(2 n-1)(2 n-3)\left(8 n^{2}-18 n+3\right) \\
& =-(2 n-3)\left(120 n^{5}-492 n^{4}+466 n^{3}+259 n^{2}-512 n+168\right) .
\end{aligned}
$$

Since we have

$$
\begin{aligned}
\left(120 n^{5}-\right. & \left.492 n^{4}+466 n^{3}+259 n^{2}\right)^{\prime \prime} \\
& =2\left(1200 n^{3}-2952 n^{2}+1398 n+259\right)>0 \text { for } n \geqq 2, \\
\left(120 n^{5}-\right. & \left.492 n^{4}+466 n^{3}+259 n-512 n\right)^{\prime} \\
& =600 n^{4}-1968 n^{3}+1398 n^{2}+518 n-512 \nearrow \text { in } 2<n<+\infty, \\
& =-28 \text { at } n=2 \text { and }=7.49875 \cdots \text { at } n=2.02
\end{aligned}
$$

and

$$
120 n^{5}-492 n^{4}+466 n^{3}+259 n^{2}-512 n+168=\left\{\begin{array}{l}
-124 \text { at } n=2, \\
-4.77919 \cdots \text { at } n=2.31, \\
5.18081 \cdots \text { at } n=2.32,
\end{array}\right.
$$

we see that

$$
D<0 \text { for } n>2.31 \cdots \text { and } D>0 \text { for } 2 \leqq n<2.31 \cdots \text {. }
$$

Therefore we see that when $n>2.31 \cdots$ we have

and so

$$
B_{4}^{\prime \prime}(x, n)>0 \text { for }-\infty<x<+\infty
$$

$$
B_{4}^{\prime}(x, n)>0 \text { and } B_{4}(x, n)>0 \text { for } 0 \leqq x<+\infty \text {. }
$$

Next, we consider the case such that $D \geqq 0$. Regarding $B_{4}{ }^{\prime \prime}(x, n) / 6$ we see

$$
0<-\frac{(2 n-3)\left(2 n^{2}-13 n+8\right)}{4(n-1)(2 n-1)(2 n-3)}=-\frac{2 n^{2}-13 n+8}{4(n-1)(2 n-1)}<1 \quad \text { for } 2 \leqq n \leqq 5
$$

and

$$
B_{4}{ }^{\prime \prime}(n, n)=6\left(8 n^{5}-20 n^{4}-2 n^{3}+23 n^{2}-3 n-3\right)
$$$$
\nearrow \text { in } 2<n<+\infty, \geqq 18 \text { for } n \geqq 2 \text {. }
$$

On the other hand, we have

and

$$
\begin{aligned}
B_{4}^{\prime}(x, n)-\frac{x}{3} & B_{4}^{\prime \prime}(x, n)=(2 n-3)\left(2 n^{2}-13 n+8\right) x^{2} \\
& +4(n-1)\left(8 n^{2}-18 n+3\right) x+n(2 n-3)(14 n-5)
\end{aligned}
$$




$$
\begin{aligned}
2(n-1)(2 n-1) & \left\{B_{4}^{\prime}(x, n)-\frac{x}{3} B_{4}{ }^{\prime \prime}(x, n)\right\}-\left(2 n^{2}-13 n+8\right) \frac{1}{6} B_{4}{ }^{\prime \prime}(x, n) \\
= & \left(120 n^{5}-492 n^{4}+466 n^{3}+259 n^{2}-512 n+168\right) x \\
& +(n-1)\left(96 n^{4}-124 n^{3}-140 n^{2}+153 n-24\right) .
\end{aligned}
$$

For the big root $x$ of $B_{4}{ }^{\prime \prime}(x, n)=0$, we obtain from the above expression

$$
\begin{aligned}
2(n-1)(2 n-1) B_{4}^{\prime}(x, n) \geqq & \left(120 n^{5}-492 n^{4}+466 n^{3}+259 n^{2}-512 n+168\right) n \\
+ & 96 n^{5}-220 n^{4}-16 n^{3}+293 n^{2}-177 n+24 \\
=120 n^{6}-396 n^{5}+ & 246 n^{4}+243 n^{3}-219 n^{2}-9 n+24,
\end{aligned}
$$

which is increasing in $2<n<+\infty$ and $\geqq 18$ for $n \geqq 2$. Hence, taking account of $B_{4}^{\prime}(0, n)>0$ for $n \geqq 2$, we have also

$$
B_{4}^{\prime}(x, n)>0 \text { for } 0 \leqq x<+\infty
$$

and so

$$
B_{4}(x, n)>0 \text { for } 0 \leqq x<+\infty \text {. }
$$

Q.E.D.

LEMMA 3.10. $B_{5}(x, n)$ has the following

i) $B_{5}(0, n)=-6 n^{2}(10 n-3)<0, B_{5}(1, n)=-36(n-1)^{2}(10 n-7)<0$,

$$
B_{5}(n, n)=n^{3}(n-1)^{2}\left(4 n^{2}-1\right)>0 \text { for } n>1 \text {. }
$$

ii) When $2 \leqq n<2.33 \cdots, B_{5}(x, n)$ is convex downward in $0<x<1$ and $\nearrow$ in $1<x<n$.

iii) When $2.33 \cdots<n \leqq 4.17, B_{5}(x, n)$ is $\searrow$ in $0<x<1$ and $\searrow$ in $1<x<n$. In ii) and iii), $2.33 \cdots$ denotes the greter root of the equation: $260 n^{2}-796 n+443$ $=0$, i.e. $(199+\sqrt{10806}) / 130=2.330399 \cdots$.

Proof. From (3.18), we obtain easily i), the discriminant of $B_{5}^{\prime \prime \prime}(x, n) / 24$ becomes

$$
\begin{gathered}
\left(20 n^{3}-60 n^{2}+139 n-107\right)^{2}+5\left(12 n^{2}-24 n+11\right)\left(20 n^{3}-240 n^{2}+367 n-102\right) \\
=400 n^{6}-1200 n^{5}-7640 n^{4}+30960 n^{3}-31199 n^{2}+2679 n+5839,
\end{gathered}
$$

which is negative for $2 \leqq n \leqq 4.17$ and positive for $4.18 \leqq n<+\infty$, as it is shown by observing its derivatives. Hence, we obtain that when $2 \leqq n \leqq 4.17$

$$
B_{5}^{\prime \prime \prime}(x, n)>0 \text { for }-\infty<x<+\infty \text {, }
$$

and $B_{5} "(x, n)$ is increasing in $-\infty<x<+\infty$.

Next, we have 


$$
\begin{gathered}
B_{5}{ }^{\prime \prime}(0, n)=-2\left(120 n^{3}-402 n^{2}+271 n-21\right) \\
>0 \text { for } 2 \leqq n \leqq 2.4 \cdots, \text { and }<0 \text { for } n>2.4 \cdots, \\
B_{5}{ }^{\prime \prime}(1, n)=-2\left(300 n^{3}-1722 n^{2}+2686 n-1189\right) \\
>0 \text { for } 2 \leqq n<3.5 \cdots, \text { and }<0 \text { for } n>3.5 \cdots, \\
B_{5}{ }^{\prime \prime}(n, n)=2\left(120 n^{5}-180 n^{4}-14 n^{3}-57 n^{2}+35 n+21\right)>0 \text { for } n \geqq 2,
\end{gathered}
$$

and

$$
\begin{gathered}
B_{5}^{\prime}(0, n)=-n\left(140 n^{2}-228 n+39\right)<0 \text { for } n \geqq 2, \\
B_{5}^{\prime}(1, n)=-520 n^{3}+2112 n^{2}-2478 n+886 \\
=-2(n-1)\left(260 n^{2}-796 n+443\right) \\
>0 \text { for } 2 \leqq n<\frac{199+\sqrt{10806}}{130}=2.330399 \cdots, \\
\text { and } \quad<0 \text { for } \frac{199+\sqrt{10806}}{130}<n<+\infty, \\
B_{5}^{\prime}(n, n)=n(n-1)\left(40 n^{4}-20 n^{3}+14 n^{2}+5 n-3\right)>0 \text { for } n \geqq 2 .
\end{gathered}
$$

Thus, we can imagine the shape of the graph of $y=B_{5}^{\prime}(x, n)$ as shown in Fig. 7 and obtain the claims ii) and iii). Finally, we obtain easily from (3.18)

$$
\begin{aligned}
& B_{5}(0, n)=-6 n^{2}(10 n-3), \\
& B_{5}(1, n)=-36(n-1)^{2}(10 n-7),
\end{aligned}
$$
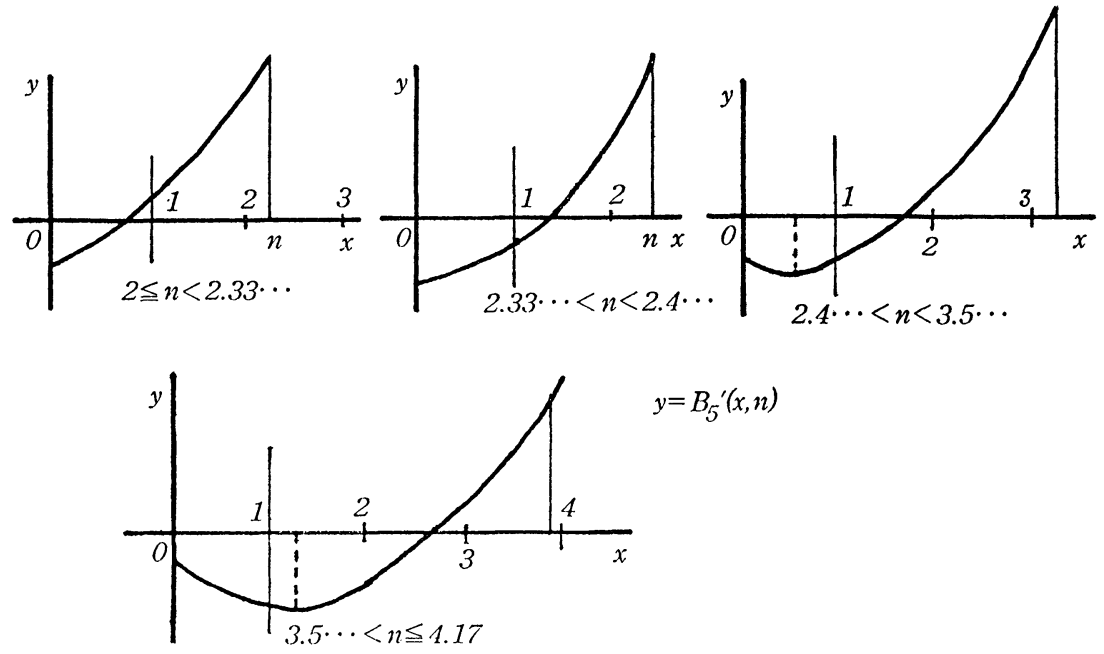

Fig. 7. 


$$
B_{5}(n, n)=n^{3}(n-1)^{2}\left(4 n^{2}-1\right) .
$$

Q.E.D.

Now, doing a long elaborate computation, we obtain

$$
\begin{aligned}
& \Pi(1+(n-1) t, n)=\frac{1}{6}(n-1)^{3}\left(10 n^{4}-25 n^{3}+13 n^{2}+17 n-6\right) i^{4} \\
& \quad+\frac{1}{60}(n-1)^{3}\left(80 n^{5}-346 n^{4}+479 n^{3}-171 n^{2}-48 n+36\right) t^{5}+\cdots
\end{aligned}
$$

and we see easily

$$
10 n^{4}-25 n^{3}+13 n^{2}+17 n-6 \geqq 40 \text { for } n \geqq 2
$$

(see Lemma 3.7) and

$$
80 n^{5}-346 n^{4}+479 n^{3}-171 n^{2}-48 n+36
$$

is increasing in $2<n<+\infty$ and $\geqq 112$ for $n \geqq 2$. Finally noticing that $(n-x)\left(\log \frac{n-x}{n-1}+\frac{x-1}{n-x}\right)$ is decreasing in $0<x<1$ and increasing in $1<x<n$ with $n>1$, since we have

$$
\frac{d}{d x}(n-x)\left(\log \frac{n-x}{n-1}+\frac{x-1}{n-x}\right)=-\log \frac{n-x}{n-1},
$$

and making use of Lemmas 3.8-3.10 and taking account of (3.19), we have investigated the sign of $\Pi(x, n)$ by a computor. We computed approximately the values of $\Pi(x, n)$ for $0 \leqq x \leqq n$ with step $1 / 100$ and for $2.2 \leqq n \leqq 5$ with step $1 / 50$, and then found out for $2.8 \leqq n \leqq 5$

$$
\Pi(x, n)>0 \text { for } w(n) \leqq x<n, x \neq 1,
$$

which can be proved by the same way as used in the previous papers, here we cite some data as follows.

$$
\begin{aligned}
& n=2.6: \Pi(0)=-44.12098, \Pi(0.49)=0.03175, \Pi(2.59)=27.05419, \\
& w(2.6)=0.38252, z(2.6)=1.73948 ; \\
& n=2.8: \Pi(0)=-55.80181, \Pi(0.48)=0.01790, \Pi(2.79)=57.55155, \\
& w(2.8)=0.49188, z(2.8)=1.60241 ; \\
& n=3.4: \Pi(0)=-103.05941, \Pi(0.47)=0.05794, \Pi(3.39)=293.78317, \\
& w(3.4)=0.68373, z(3.4)=1.36120 ; \\
& n=5: \Pi(0)=-344.70200, \Pi(0.45)=0.09335, \Pi(4.99)=4169.85710, \\
& w(5)=0.86576, z(5)=1.14391 .
\end{aligned}
$$


Thus, we see that

$$
\Gamma(x, n) \text { is increasing in } w(n)<x<n \text {, with } 2.8 \leqq n \leqq 5 .
$$

Then, we compute approximately the values of $\Gamma(x, n)$ in order to prove (3.9) for $\frac{5+\sqrt{13}}{4}+\frac{11}{1000} \leqq n \leqq 2.8$. First, regarding the expressions in (3.10) we have the following

LEMMA 3.11. Setting $m=(5+\sqrt{13}) / 4=2.15138 \cdots$, we have:

i) $4(4 n+1) x^{2}-5\left(4 n^{2}+14 n-7\right) x+80 n^{2}-48 n+3$

is decreasing in $0<x<2.5$ and $\geqq 0.38443$

$$
\text { for } 0 \leqq x \leqq m \text { with } n \geqq m+0.011 \text {. }
$$

ii) $-2(4 n-5) x^{3}+\left(10 n^{2}-31 n+23\right) x^{2}+\left(20 n^{2}-54 n+9\right) x$

$+3 n(10 n-3)$ is positive for $0 \leqq x \leqq m$ with $n \geqq 2$

and $\searrow \nearrow$ in $0<x<m$ with $2 \leqq n<2.521 \cdots$

and $\nearrow \searrow$ in $0<x<m$ with $2.521 \cdots<n \leqq 3.66 \cdots(2.521 \cdots=(27+3 \sqrt{61}) / 20)$.

iii) $\tilde{Q}_{2}(x, n)=(n-1)(2 n-3) x^{2}+\left(4 n^{2}-10 n+3\right) x+3 n(2 n-1)$

is increasing in $0<x<+\infty$ with $n \geqq m$.

Proof. i) We see easily that

$$
\frac{5\left(4 n^{2}+14 n-7\right)}{8(4 n+1)}>2.5 \text { for } n \geqq 2,
$$

hence this polynomial of $x$ is decreasing in $0<x<2.5$. Since we have $4 m^{2}-10 m+3$ $=0$, at $x=m$ this polynomial is equal to

$$
\begin{aligned}
& (80-20 m) n^{2}-30(m+2) n+45 m \\
& >36.97224 n^{2}-124.54164 n+96.81245>0.38443 \text { for } n \geqq m+0.011 .
\end{aligned}
$$

ii) Regarding the derivative of the cubic polynomial $P$ of $x$ :

$$
Q=-6(4 n-5) x^{2}+2\left(10 n^{2}-31 n+23\right) x+20 n^{2}-54 n+9,
$$

we see that its discriminant $>0$ for $n \geqq 2$,

$$
\begin{gathered}
\frac{10 n^{2}-31 n+23}{6(4 n-5)} \text { is increasing in } 2<n<+\infty, \\
=\frac{1}{18} \text { at } n=2, \frac{10}{21} \text { at } n=3, \frac{59}{66} \text { at } n=4,
\end{gathered}
$$


and $Q$ becomes at $x=2$

$$
60 n^{2}-274 n+221 \leqq-3 \text { for } 2 \leqq n \leqq 3.5 \text {, }
$$

and hence it is negative at $x=m$ when $2 \leqq n \leqq 3.5$, and

$$
\begin{aligned}
20 n^{2}-54 n+9 & <0 \text { for } 2<n<\frac{27+3 \sqrt{61}}{20}=2.52153 \cdots, \\
& >0 \text { for } \frac{27+3 \sqrt{61}}{20}<n<+\infty .
\end{aligned}
$$

By solving the quadratic equation on $n$ :

$$
-6(4 n-5) m^{2}+2\left(10 n^{2}-31 n+23\right) m+20 n^{2}-54 n+9=0,
$$

we get the bigger root $n=3.66786 \cdots$. Next, we compute the value of $P$ at $x=m$ as follows. Using $4 m^{2}=10 m-3$, we have

$$
\begin{aligned}
&-2(4 n-5) m^{3}+\left(10 n^{2}-31 n+23\right) m^{2}+\left(20 n^{2}-54 n+9\right) m+30 n^{2}-9 n \\
&=(45 m+22.5) n^{2}-(175.5 m-29.25) n+121.5 m-36 \\
& \geqq 4(45 m+22.5)-2(175.5 m-29.25)+121.5 m-36 \\
&=-49.5 m+112.5=6.00630 \cdots>0 \quad \text { for } n \geqq 2 .
\end{aligned}
$$

Hence, from these facts we see that this cubic polynomial $P$ is positive for $0 \leqq x \leqq m$, with $n \geqq(27+3 \sqrt{61}) / 20=2.52 \cdots$. When $2 \leqq n<(27+3 \sqrt{61}) / 20$, we have to invextigate its sign at $x=x_{0}$ : the smaller roof of

$$
-6(4 n-5) x^{2}+2\left(10 n^{2}-31 n+23\right) x+20 n^{2}-54 n+9=0 .
$$

Since we have

$$
\begin{gathered}
3 P-x Q=\left(10 n^{2}-31 n+23\right) x^{2}+2\left(20 n^{2}-54 n+9\right) x+90 n^{2}-27 n \\
6(4 n-5)(3 P-x Q)+\left(10 n^{2}-31 n+23\right) Q \\
>2\left(100 n^{4}-140 n^{3}-475 n^{2}+410 n+259\right) x \\
+200 n^{4}+1000 n^{3}-1124 n^{2}-711 n+207
\end{gathered}
$$

and

and

$$
\begin{gathered}
100 n^{4}-140 n^{3}-475 n^{2}+410 n+259 \text { is increasing in } 2<n<+\infty, \\
=-13.82063 \cdots \text { at } n=2.47 \text { and } 1.68313 \cdots \text { at } n=2.48,
\end{gathered}
$$

$$
0<x_{0}<\frac{10 n^{2}-31 n+23}{6(4 n-5)} \leqq \frac{7.624}{29.52}=0.25826 \cdots<0.26,
$$

for $2 \leqq n \leqq 2.48$, we obtain 


$$
\begin{aligned}
& {[18(4 n-5) P]_{x=x_{0}}>2 \times 0.26\left(100 n^{4}-140 n^{3}-475 n^{2}+410 n+259\right)} \\
& \quad+200 n^{4}+1000 n^{3}-1124 n^{2}-711 n+207 \\
& =252 n^{4}+927.2 n^{3}-1371 n^{2}-497.8 n+341.68>0 \text { for } 2 \leqq n \leqq 2.47 \cdots .
\end{aligned}
$$

From these facts, we can show the graph of $y=P(x, n)$ as Fig. 8 .

iii) is easily checked from the expression of $\tilde{Q}_{2}(x, n)$ Q. E.D.
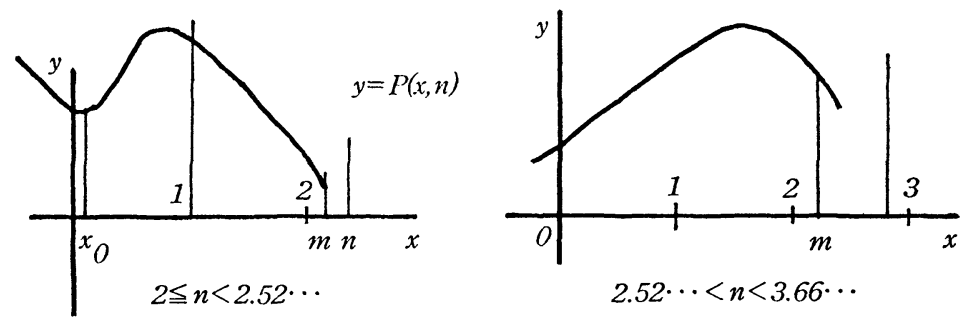

Fig. 8.

Then, we computed approximately the values of $\Gamma(x, n)$ for $0 \leqq x \leqq n$ with step $1 / 100$ and for $2.15 \leqq n \leqq 2.19$ with step $1 / 100$ and for $2.2 \leqq n \leqq 2.9$ with step $1 / 10$ and find out

$$
\Gamma(w(n), n)<\Gamma(Z(n), n) \text { for } 2.152 \leqq n \leqq 2.8,
$$

which can be proved, using Lemma 3.11 and developing a special method for $n$ near $\frac{5+\sqrt{13}}{4}$, which is ommited here to describe, since it needs a large space. Thus, we obtain the following

PROPOSITION 8. $Z(n)$ is decreasing in $2.152 \leqq n \leqq 22$.

$\S 4$. Positiveness of $\eta\left(X_{n}(x)\right)-\eta(x, n)$ and $V\left(x, X_{n}(x)\right)$.

Noticing the arguments developed in $\S 3$, we define inductively a series of constants $z_{i}(n)$ and $w_{i}(n), i=1,2, \cdots$, as follows:

$$
\left\{\begin{array}{l}
z_{1}(n):=z(n), \quad w_{1}(n):=w(n), \\
z_{2}(n):=Z(n), \quad w_{2}(n):=X_{n}^{-1}\left(z_{2}(n)\right)
\end{array}\right.
$$

and, supposing $z_{i}(n)$ and $w_{i}(n)$ are defined for $i>2$ as

$$
\left\{\begin{array}{l}
z_{\imath}(n)>z_{2}(n)>\cdots>z_{i}(n)>1, \\
w_{1}(n)<w_{2}(n)<\cdots<w_{i}(n)<1,
\end{array}\right.
$$


$z_{\imath+1}(n)$ and $w_{\imath+1}(n)$ are determined by

$$
\left\{\begin{array}{l}
\eta\left(z_{\imath+1}(n), n\right)=\eta\left(w_{i}(n), n\right) \quad \text { with } 1<z_{\imath+1}(n)<n, \\
w_{\imath+1}(n):=X_{n}^{-1}\left(z_{\imath+1}(n)\right) .
\end{array}\right.
$$

When $n>(1+\sqrt{13}) / 2=2.30277 \cdots$, by means of Proposition 1 , if $w_{i}(n)$ satisfies

$$
\eta\left(w_{i}(n), n\right)>-\frac{n(n-1)^{n-2}}{2 \sqrt{n-1}},
$$

then $z_{\imath+1}(n)$ can be defined by (4.3) and

$$
1<z_{\imath+1}(n)<z_{i}(n),
$$

because $0<w_{2-1}(n)<w_{i}(n)<\alpha(n)$ and the behavior of $\eta(x, n)$ as shown in Fig. 3, and

$$
1>w_{\imath+1}(n)>w_{\imath}(n),
$$

which is evident from (4.3) and the property of $X_{n}(x)$. Considering these facts, we continue the above process to define $z_{\imath+2}(n)$ and $w_{\imath+2}(n)$ as long as (4.4) is satisfied for $i+1$.

PROPOSITION 9. If $z_{i}(n)$ and $w_{i}(n), i \geqq 1$, are defined in some interval of $n>(1+\sqrt{13}) / 2$, then $z_{i}(n)$ is decreasing and $w_{\imath}(n)$ increasing there.

Proof. $z_{1}(n), w_{1}(n)$ and $z_{2}(n)$ have these properties in $(5+\sqrt{13}) / 4+0.011 \leqq$ $n \leqq 22$ by Propositions 4,5 and 8 . Observing the arguments in the proofs of these propositions, we see that the arguments are also available for $z_{i}(n)$ and $w_{i}(n), i \geqq 2$. Hence the statement is true.

Q.E.D.

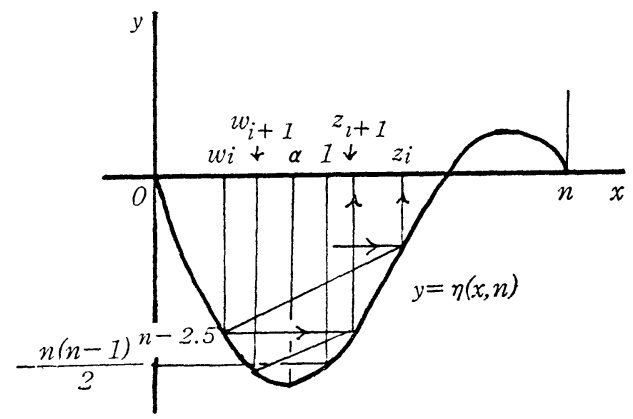

Fig. 9.

Proposition 10. When $n>(1+\sqrt{1 \overline{3}}) / 2$, we have

$$
\eta\left(X_{n}(x), n\right)-\eta(x, n)>0 \text { for } 0<x<1 .
$$


Proof. If $w_{1}(n) \geqq \alpha(n)$, the above inequality holds by Lemma 3.3. So, we suppose $w_{1}(n)<\alpha(n)$ and

$$
\eta\left(w_{1}(n), n\right) \leqq-\frac{n(n-1)^{n-2}}{2 \sqrt{n-1}}
$$

then

$$
\eta\left(X_{n}(x), n\right)-\eta(x, n)>0
$$

for $0<x<w(n)$, since we have

$$
\eta(x, n)<0<\eta\left(X_{n}(x), n\right) .
$$

Next, we suppose

$$
\eta\left(w_{i}(n), n\right)>-\frac{n(n-1)^{n-2}}{2 \sqrt{n-1}}
$$

and

$$
\eta\left(w_{\imath+1}(n), n\right) \leqq-\frac{n(n-1)^{n-2}}{2 \sqrt{n-1}}
$$

for a fixed $i>1$. Then, we see $w_{i}(n)<\alpha(n)$ and

$$
\eta\left(X_{n}(x), n\right)-\eta(x, n)>0 \quad \text { for } \quad w_{i}(n) \leqq x \leqq w_{\imath+1}(n) .
$$

And by (4.2) it holds also for $0<x \leqq w_{\imath}(n)$. Next, for $w_{\imath+1}(n)<x<1$, we have

$$
\eta\left(X_{n}(x), n\right)>-\frac{n(n-1)^{n-2}}{2 \sqrt{n-1}}>\eta(x, n) .
$$

Q.E.D.

The length of series of $z_{i}(n)$ and $w_{i}(n)$ is depend on $n$. For each $n>$ $(1+\sqrt{13}) / 2$ we determine $j(n)$ as follows: If

$$
\eta\left(w_{1}(n), n\right) \leqq-\frac{n(n-1)^{n-2}}{2 \sqrt{n-1}},
$$

then $j(n)=1$, otherwise $j(n)=i+1$ by

$$
\eta\left(w_{i}(n), n\right)>-\frac{n(n-1)^{n-2}}{2 \sqrt{n-1}} \text { and } \eta\left(w_{\imath+1}(n), n\right) \leqq-\frac{n(n-1)^{n-2}}{2 \sqrt{n-1}} .
$$

We computed the values of $z_{i}(n), w_{\imath}(n)$ by a main frame computor and found, for example, 


\begin{tabular}{cc|cc|cc}
$n$ & $j(n)$ & $n$ & $j(n)$ & $n$ & $j(n)$ \\
\hline 2.303 & 81 & 2.38 & 6 & 2.47 & \\
.310 & 30 & .39 & 6 & $\vdots$ & 3 \\
.32 & 18 & 2.40 & 5 & 2.55 & \\
.33 & 13 & .41 & 5 & 2.56 & \\
.34 & 11 & .42 & 5 & $\vdots$ & 2 \\
.35 & 9 & 2.43 & & 2.80 & \\
.36 & 8 & $\vdots$ & 4 & 2.90 & \\
.37 & 7 & 2.46 & & $\vdots$ & 1 \\
& & & & 5.00 &
\end{tabular}

Now, we try to prove the positiveness of $V\left(x, X_{n}(x)\right)$, by using numerical approximation by computors. Regarding the expression of $V\left(x, X_{n}(x)\right)$ by (1.13), we have studied $\eta(x, n)$ thus far. We know the following facts: i) $\frac{x^{2} \sqrt{n-x}}{(1-x)^{5}}$ is increasing in $0<x<1$ with $n>1$ (Lemma 3.2 in (VII)); ii) $F_{2}(x)$ is decreasing in $0<x<1$ with $n>2$ and positive there (Lemma 3.1 in (VII)).

LEMMA 4.1. When $n \geqq 2, \tilde{\lambda}\left(X_{n}(x)\right)-\tilde{\lambda}(x)$ is decreasing in $0<x<1$ and positive there and

$$
\lim _{x \rightarrow+0}\left\{\tilde{\lambda}\left(X_{n}(x)\right)-\tilde{\lambda}(x)\right\}=+\infty .
$$

Proof. Setting $X_{n}(x)=X(x)$, we have

$$
\begin{aligned}
\frac{d}{d x} & \{\tilde{\lambda}(X(x))-\tilde{\lambda}(x)\} \\
& =-\frac{(X-1)\{n+(n-1) X\}}{(n-1) X^{2}(n-X)} \frac{d X}{d x}+\frac{(x-1)\{n+(n-1) x\}}{(n-1) x^{2}(n-x)}
\end{aligned}
$$

by Lemma 7.1 in (III) and

$$
\frac{d X}{d x}=\frac{1-x}{x(n-x)} \cdot \frac{X(n-X)}{1-X},
$$

hence

$$
\begin{aligned}
& \frac{d}{d x}\{\tilde{\lambda}(X(x))-\tilde{\lambda}(x)\} \\
& \quad=\frac{n(1-x)}{(n-1) x(n-x)}\left(\frac{1}{X}-\frac{1}{x}\right)<0 \text { for } 0<x<1 .
\end{aligned}
$$

Since we have

$$
\lim _{x \rightarrow 1}\left\{\tilde{\lambda}\left(X_{n}(x)\right)-\tilde{\lambda}(x)\right\}=0,
$$

this function is positive for $0<x<1$. 
Next, we have from (1.8)

$$
\begin{aligned}
\lim _{x \rightarrow+0} & \{\tilde{\lambda}(X(x))-\tilde{\lambda}(x)\} \\
& =\lim _{x \rightarrow+0}\left\{\log (n-X)-\frac{1}{(n-1) X}-\log (n-x)+\frac{1}{(n-1) x}\right\} \\
& =\lim _{x \rightarrow+0}\left\{\log (n-X)+\frac{1}{(n-1) x}\right\}-\frac{1}{n(n-1)}-\log n, X=X_{n}(x) .
\end{aligned}
$$

From $x(n-x)^{n-1}=X(n-X)^{n-1}$, we have

$$
\begin{aligned}
\log ( & n-X)+\frac{1}{(n-1) x} \\
= & \left\{\log (n-x)+\frac{1}{n-1}(\log x-\log X)\right\}+\frac{1}{(n-1) x} \\
& =\frac{1}{n-1} \frac{x \log x+1}{x}+\log (n-x)-\frac{1}{n-1} \log X,
\end{aligned}
$$

hence we have

$$
\lim _{x \rightarrow+0}\left\{\log (n-X)+\frac{1}{(n-1) x}\right\}=\frac{n-2}{n-1} \log n+\lim _{x \rightarrow+0} \frac{x \log x+1}{x}=+\infty,
$$

from which we obtain

$$
\lim _{x \rightarrow+0}\left\{\tilde{\lambda}\left(X_{n}(x)\right)-\tilde{\lambda}(x)\right\}=+\infty
$$

Q.E.D.

Taking account of these facts, we computed approximately the values of $V\left(x, X_{n}(x)\right) /(n-1)^{n-1}$ for $0<x<1$ with step $1 / 100$ and $2.30 \leqq n \leqq 5.00$ with step $1 / 50$ by a computor and found that

$$
V\left(x, X_{n}(x)\right)<0
$$

for some interval of $x$ as follows:

$$
\begin{aligned}
n=2.30: & 0.20----0.99 \\
2.32: & 0.25----0.96 \\
2.34: & 0.29----0.91 \\
2.36: & 0.35----0.85 \\
2.38: & 0.43----0.77
\end{aligned}
$$

and when $2.40 \leqq n \leqq 5.00$

$$
V\left(x, X_{n}(x)\right)>0 \text { for } 0<x<1,
$$

which can be proved by the same ways which have been used for the verifications of certain propositions in (VI)-(VIII), in fact, we have prepared so far also many lemmas and propositions for this purpose. 
Combining the above result with the ones in (IV)-(VIII), we obtain the main theorem of this paper as follows.

THEOREM C. The period $T$ as a function of $\tau=\left(x_{1}-1\right) /(n-1)$ and $n$ is monotone decreasing with respect to $n \geqq 2.4$ for any fixed $\tau(0<\tau<1)$.

Remark. By the fact disclosed in this paper, we see that, to prove Theorem C by means of (4.4), $n=2.4$ is nearly the lower limit of the available interval of $n$. Therefore, we have to develope completely different methods from the ones used until now for $2 \leqq n<2.4$.

At last, we cite the table of the constants used in the arguments in the present paper.

Table A

\begin{tabular}{ccccc}
$n$ & $\alpha$ & $w$ & $z$ & $Z$ \\
\hline 2.16 & 1.136433 & 0.003389 & 2.151726 & 2.146321 \\
2.17 & 1.125524 & 0.009526 & 2.148953 & 2.136309 \\
2.18 & 1.114824 & 0.016968 & 2.144188 & 2.123337 \\
2.19 & 1.104334 & 0.025294 & 2.137989 & 2.108150 \\
2.20 & 1.094053 & 0.034253 & 2.130695 & 2.091227 \\
2.21 & 1.083979 & 0.043671 & 2.122543 & 2.072922 \\
2.22 & 1.074113 & 0.053421 & 2.113711 & 2.053505 \\
2.23 & 1.064450 & 0.063405 & 2.104338 & 2.033198 \\
2.24 & 1.054989 & 0.073547 & 2.094537 & 2.012180 \\
2.25 & 1.045726 & 0.083788 & 2.084398 & 1.990601 \\
& & & & \\
2.26 & 1.036659 & 0.094080 & 2.073995 & 1.968586 \\
2.27 & 1.027785 & 0.104383 & 2.063391 & 1.946244 \\
2.28 & 1.019098 & 0.114666 & 2.052638 & 1.923664 \\
2.29 & 1.010597 & 0.124905 & 2.041781 & 1.900926 \\
2.30 & 1.999410 & 0.135077 & 2.030856 & 1.878095 \\
2.31 & 0.994133 & 0.145167 & 2.019895 & 1.855231 \\
2.32 & 0.986165 & 0.155161 & 2.008925 & 1.832382 \\
2.33 & 0.978365 & 0.165048 & 1.997968 & 1.809591 \\
2.34 & 0.970731 & 0.174819 & 1.987044 & 1.786895 \\
2.35 & 0.963259 & 0.184468 & 1.976170 & 1.764327 \\
2.36 & 0.955944 & 0.193988 & 1.965360 & 1.741912 \\
2.37 & 0.948783 & 0.203377 & 1.954625 & 1.719676 \\
2.38 & 0.941773 & 0.212631 & 1.943976 & 1.697637 \\
2.39 & 0.934909 & 0.221748 & 1.933421 & 1.675812 \\
2.40 & 0.928188 & 0.230726 & 1.922968 & 1.654217 \\
2.41 & 0.921607 & 0.239565 & 1.912623 & 1.632864 \\
2.42 & 0.915161 & 0.248265 & 1.902390 & 1.611761 \\
& & & &
\end{tabular}




$\begin{array}{lllll}2.43 & 0.908847 & 0.256827 & 1.892274 & 1.590919 \\ 2.44 & 0.902663 & 0.265249 & 1.882278 & 1.570343 \\ 2.45 & 0.896604 & 0.273535 & 1.872405 & 1.550039 \\ 2.46 & 0.890667 & 0.281684 & 1.862656 & 1.530012 \\ 2.47 & 0.884850 & 0.289699 & 1.853034 & 1.510264 \\ 2.48 & 0.879150 & 0.297581 & 1.843538 & 1.490797 \\ 2.49 & 0.873562 & 0.305332 & 1.834171 & 1.471613 \\ 2.50 & 0.868085 & 0.312954 & 1.824932 & 1.452713 \\ 2.6 & 0.818805 & 0.382522 & 1.739489 & 1.279068 \\ 2.7 & 0.777915 & 0.441509 & 1.665843 & 1.131452 \\ 2.8 & 0.743593 & 0.491885 & 1.602412 & 1.006263 \\ 2.9 & 0.714475 & 0.535272 & 1.547598 & 1.000051 \\ 3.0 & 0.689531 & 0.572949 & 1.500000 & 1.000040 \\ & & & & \\ 3.1 & 0.667975 & 0.605919 & 1.458442 & 1.000038 \\ 3.2 & 0.649199 & 0.634976 & 1.421960 & 1.000030 \\ 3.3 & 0.632727 & 0.660745 & 1.389763 & \\ 3.4 & 0.618184 & 0.683731 & 1.361204 & \\ 3.5 & 0.605268 & 0.704341 & 1.335751 & \\ 3.6 & 0.593735 & 0.722906 & 1.312964 & \\ 3.7 & 0.583388 & 0.739702 & 1.292480 & \\ 3.8 & 0.574062 & 0.754955 & 1.273994 & \\ 3.9 & 0.565621 & 0.768857 & 1.257251 & \\ 4.0 & 0.557953 & 0.781570 & 1.242034 & \\ 4.1 & 0.550963 & 0.793229 & 1.228162 & \\ 4.2 & 0.544568 & 0.803953 & 1.215476 & \\ 4.3 & 0.538702 & 0.813842 & 1.203845 & \\ 4.4 & 0.533303 & 0.822982 & 1.193151 & \\ 4.5 & 0.528323 & 0.831451 & 1.183296 & \\ 4.6 & 0.523717 & 0.839313 & 1.174193 & \\ 4.7 & 0.519447 & 0.846627 & 1.165765 & \\ 4.8 & 0.515479 & 0.853444 & 1.157946 & \\ 4.9 & 0.511785 & 0.859809 & 1.150679 & \\ 5.0 & 0.508339 & 0.865761 & 1.143911 & \\ 2.84 & 0.731385 & 0.510000 & 1.579536 & 1.000070 \\ 3.24 & 0.642357 & 0.645647 & 1.408606 & \\ & & & & \\ & & \end{array}$

$$
\begin{aligned}
& \frac{5+\sqrt{13}}{4}=2.151387819 \cdots, \\
& \frac{1+\sqrt{13}}{2}=2.302775638 \cdots
\end{aligned}
$$




\section{REFERENCES}

[1] S. S. Chern, M. do Carmo and S. Kobayashi, Minimal Submanifolds of a sphere with second fundamental form of constant length, Functional Analysis and Related Fields, Springer-Verlag, 1970, 60-75.

[2] S. FURUYA, On periods of periodic solutions of a certain nonlinear differential equation, Japan-United States Seminar on Ordinary Differential and Functional Equations, Lecture Notes in Mathematics, Springer-Verlag, 243 (1971), 320-323.

[3] W.Y. Hsiang and H. B. Lawson, JR., Minimal submanifolds of low cohomogeneity, J. Diff. Geometry, 5 (1970), 1-38.

[4] T. Otsuki, Minimal hypersurfaces in a Riemannian manifold of constant curvature, Amer. J. Math., 92 (1970), 145-173.

[5] T. Otsuki, On integral inequalities related with a certain nonlinear differential equation, Proc. Japan Acad., 48 (1972), 9-12.

[6] T. Otsuki, On a 2-dimensional Riemannian manifold, Differential Geometry, in honor of K. Yano, Kinokuniya, Tokyo, 1972, 401-414.

[7] T. Otsuki, On a family of Riemannian manifolds defined on an $m$-disk, Math. J. Okayama Unıv., 16 (1973), 85-97.

[8] T. Отsuki, On a bound for periods of solutions of a certain nonlinear differential equation (I), J. Math. Soc. Japan, 26 (1974), 206-233.

[9] T. Отsuki, On a bound for periods of solutions of a certain nonlinear differential equation (II), Funkcialaj Ekvacioj., 17 (1974), 193-205.

[10] T. Oтsuki, Geodesics of $O_{n}^{2}$ and an analysis on a related Riemann surface, Tôhoku Math. J., 28 (1976), 411-427.

[11] T. Otsuki, A certain property of geodesics of the family of Riemannian manifolds $O_{n}^{2}(\mathrm{I})$, Proc. of Japan-United States Seminar on Minimal Submanifolds, including Geodesics, Kaigai, Tokyo, 1978, 173-192.

[12] T. Otsuki, A certain property of geodesics of the family of Riemannian manifolds $O_{n}^{2}$ (II), Kodai Math. J., 2 (1979), 211-242.

[13] T. Otsuki, A certain property of geodesics of the family of Riemannian manifolds $O_{n}^{2}$ (III), Kodai Math. J., 4 (1981), 28-70.

[14] T. Otsuki, A certain property of geodesics of the family of Riemannian manifolds $O_{n}^{2}$ (IV), Kodai Math. J., 5 (1982), 160-199.

[15] T. OTsukI, A certain property of geodesics of the family of Riemannian manifolds $O_{n}^{2}(\mathrm{~V})$, Kodai Math. J., 5 (1982), 454-481.

[16] T. OTsuki, A certain property of geodesics of the family of Riemannian manifolds $O_{n}^{2}$ (VI), Kodai Math. J., 7 (1984), 326-364.

[17] T. OTsukI, Certain inequalities related with a non-linear differential equation (I), (II), TRU Math., 20 (1984), 23-68, 69-89.

[18] T. OTSUKI, A certain property of geodesics of the family of Riemannian manifolds $O_{n}^{2}$ (VII), Kodai Math. J., 8 (1985), 375-419.

[19] T. OTsukI, Certain inequalities and constants related with a non-linear differential equation, TRU Math., 21 (1985), 5-35.

[20] T. OTsUKI, A certain property of geodesics of the family of Riemannian manifolds $O_{n}^{2}$ (VIII), Kodai Math. J., 9 (1986), 272-307.

[21] T. OTsukI, Certain inequalities and constants related with a non-linear differential equation (II), TRU Math., 21 (1985), 241-268.

[22] T. Otsuki, Certain inequalities and constants related with a non-linear differential equation (III), TRU Math., 23 (1987), 197-240. 
[23] T. Otsuki, A family of 2-dimensional Riemannian manifolds joining the noneuclidean planes, TRU Math., 17 (1981), 129-139.

[24] M. MAeda AND T. OTsuki, Models of the Riemannian manifolds $O_{n}^{2}$ in the Lorentzian 4-space, J. Diff. Geometry, 9 (1974), 97-108.

[25] M. URABE, Computations of periods of a certain nonlinear autonomous oscilations, Study of algorithms of numerical computations, Sūrikaiseki Kenkyūsho Kōkyūroku, 149 (1972), 111-129 (Japanese).

Department of Mathematics

SCIENCE UNIVERSITY OF TOKYO

WaKamiYa-cho 26, ShinjuKU-KU

TOKYO, JAPAN 162 